\title{
Screening of promising chemotherapeutic candidates from plants extracts
}

\author{
Junei Kinjo $^{1} \cdot$ Daisuke Nakano $^{1} \cdot$ Toshihiro Fujioka $^{1} \cdot$ Hikaru Okabe $^{1}$
}

Received: 29 February 2016/Accepted: 25 March 2016/Published online: 16 April 2016

(C) The Japanese Society of Pharmacognosy and Springer Japan 2016

\begin{abstract}
Over the course of our studies investigating anti-proliferative properties of compounds originating from plants against human gastric adenocarcinoma (MK-1), human uterine carcinoma (HeLa), murine melanoma (B16F10), and two human T cell lymphotropic virus type 1 (HTLV-1)-infected T-cell lines (MT-1 and MT-2), we have screened 582 extracted samples obtained from a variety of parts from 370 plants. A few extracts showed anti-proliferative activity against all cell lines, but upon further investigation, toxicity toward selected cell lines was recognized. After activity-guided fractionation, isolation of the active principles was achieved. Structure-activity relationship studies identified the components and functionalities responsible for the specific selectivity against each cancer cell line. The effect of polyacetylenes against MK-1 cells was more potent than against HeLa and B16F10 cells. The compound having a 3,4-dihydroxyphenethyl group also showed an anti-proliferative effect against B16F10 cells. Some 6-methoxyflavone derivatives and 8-hydroxy furanocoumarins were good inhibitors of HeLa cell growth. The 17 compounds whose $\mathrm{EC}_{50}$ values were less than $1 \mathrm{nM}$ did not show specific cellular selectivity. Because the cytotoxic effect of 24, 25-dihydrowithanolide D toward control cells was observed at a concentration about 100 times higher than those for the cancer cell lines, withanolide was identified as the most promising chemotherapeutic candidate in our experiments.
\end{abstract}

Junei Kinjo

kinjojun@fukuoka-u.ac.jp

1 Faculty of Pharmaceutical Sciences, Fukuoka University, 819-1 Nanakuma, Jonan-ku, Fukuoka 814-0180, Japan
Keywords Cancer cell lines - Anti-proliferative activity · Activity-guided fractionation - Plant extracts - Active principles $\cdot$ Structure-activity relationship

\section{Introduction}

Development of anti-neoplastic drugs is the focus of numerous research programs around the world. Plants are the richest source of novel chemical compounds and in fact, many natural product-derived compounds have been identified as chemotherapeutic candidates [1]. For instance, vinca alkaloids, podophyllotoxins, taxanes, and camptothecins are four main classes of compounds that are wellknown anti-neoplastic drugs originating from plants [2]. It is significant that over $60 \%$ of the currently used antineoplastic drugs are derived from natural sources including plants [3].

Over the course of our studies investigating the antiproliferative characteristics of compounds originating from plants against human gastric adenocarcinoma (MK-1), human uterine carcinoma (HeLa), murine melanoma (B16F10), and two human T-cell lymphotropic virus type 1 (HTLV-1)-infected T-cell lines (MT-1 and MT-2), we have already reported many compounds active against cancer cell lines [4]. Herein, we report not only the screening results against the above cell lines but also the active principles and analysis of their structure-activity relationships.

\section{Screening results}

The 582 samples obtained from a variety of plant parts from 370 plants (302 genera, 104 families) were extracted with $\mathrm{MeOH}$ under reflux. The anti-proliferative effects of 
the extracts against the MK-1, HeLa, B16F10, MT-1, and MT-2 cell lines were evaluated (Table 1 ). The extracts listed in Table 1 are classified in the Angiosperm Phylogeny Group III system. The extracts of the leaves of Annona squamosa (Annonaceae), the aerial parts and roots of Tylophora tanakae (Asclepiadaceae), and the leaves of Thuja occidentalis (Cupressaceae) showed the most potent anti-proliferative activities against all cell lines. The extracts of the leaves of Annona cherimola (Annonaceae), the fresh leaves of Tylophora ovata and T. ovata var. brownii (Asclepiadaceae), twigs of T. ovata, the roots of Saussurea lappa (Asteraceae), the seeds of Luffa acutangula (Cucurbitaceae), the leaves of Juniperus rigida (Cupressaceae), the woods of Haematoxylum brasileto (Fabaceae), the rhizomes of Coptis japonica (Ranunculaceae), the roots of Ruta graveolens (Rutaceae), and the leaves of Cephalotaxus harringtonia (Taxaceae) showed decreasing levels of potency in the order listed. Homoharringtonine (Omacetaxine), a protein translation inhibitor for the treatment of chronic myelogenous leukemia, is found in the leaves of $C$. harringtonia [5]; therefore, the extract might show potent activity. There were a few extracts that had anti-proliferative activity against all cell lines and upon further investigation, toxicity toward selected cell lines was identified.

Table 2 shows a summary of the sensitivity of the plant extracts toward MK-1, HeLa, B16F10, MT-1, and MT-2 cells. The percentage of extracts that were active at concentrations of less than $100 \mu \mathrm{g} / \mathrm{mL}$ against the various cell lines were as follows: B16F10 (70 \%), MK-1 (55\%), HeLa (39\%), MT-1 (23\%), and MT-2 (28\%). Adult T-cell leukemia/lymphoma (ATL) is a malignancy of mature peripheral $\mathrm{T}$ lymphocytes caused by HTLV-1. Although conventional chemotherapeutic regimens used against other malignant lymphomas have been administered to ATL patients, the therapeutic outcomes remain very poor. Therefore, these results suggest that a few plant extracts were sensitive to the T-cell lymphotropic virus type 1 (HTLV-1)-infected T cells (MT-1 and MT-2).

\section{Active principles}

\section{Polyacetylenes (Fig. 1)}

After activity-guided fractionation against MK-1 cells, two active polyacetylenes, falcarindiol (1) and panaxynol (2), were isolated from the roots of Heracleum moellendorffii (Apiaceae) [6]. Six other polyacetylenes were isolated from the roots of Angelica japonica (Apiaceae) [7] together with $\mathbf{1}$ and $\mathbf{2}$ after activity-guided fractionation against MK-1 cells. Among them, four compounds were furanocoumarin ethers of 1. It was evident that the effects of these compounds except for compound 3 against MK-1 cells were more potent than their effects against HeLa and B16F10 cells (Table 3 ). Because compound 2 showed 16 times greater activity when compared with its 8-hydroxy derivative (1), the presence of a hydroxy group at C- 8 was presumed to reduce activity. The most potent compound was panaxynol (2), with an $\mathrm{EC}_{50}$ value of $1.2 \mu \mathrm{M}$ against MK-1 cells. Bioactive panaxynol-type polyacetylenes in plant-derived foods have attracted attention as health-promoting compounds [8].

\section{Lignans (Fig. 2)}

After activity-guided fractionation against MK-1, HeLa, and B16F10 cells, seven lignans including deoxypodophyllotoxin (9), (-)-deoxypodorhizone (10), and related compounds were isolated from the roots of Anthriscus sylvestris (Apiaceae) [9]. From the fruits of the same plant, two other lignans (14 and 15) were isolated together with 9 and $\mathbf{1 0}$ after activity-guided fractionation against MK-1, HeLa, and B16F10 cells [10]. Deoxypodophyllotoxin (9) showed higher activity than polyacetylenes against these cell lines. Etoposide, a clinically used chemotherapeutic agent against small-cell lung cancer, malignant lymphoma, and acute leukemia is a derivative of a podophyllotoxin isolated from Podophyllum peltatum (Berberidaceae) [11]. Of note is that the $\mathrm{EC}_{50}$ value of deoxypodophyllotoxin (9) was in the nanomolar range across all cell lines tested including MT-1 and MT-2 cells (Table 3). Topoisomerase II-inhibited DNA breakage was recognized as the mechanism of action of Etoposide. The structural features that are crucial for the anti-topoisomerase II activity of podophyllotoxin derivatives have been roughly identified as: bulky $7 \beta$-bulky substituent, trans-lactone in ring $\mathrm{D}$, dioxolane ring in ring $\mathrm{A}$, quasi-axial configuration of ring $\mathrm{E}$, and 4 'hydroxy group [12].

\section{Phenylethanoids (Fig. 3)}

After activity-guided fractionation against B16F10 cells, two active phenylethanoids, acteoside (17) and isoacteoside (18), were isolated from the leaves of Clerodendrum bungei and the bark of C. trichotomum (Laminaceae) [13]. Four other phenylethanoids including arenarioside (19) and leucosceptoside A (20) were isolated from the aerial parts of Lippia dulcis and L. canescens (Verbenaceae) together with some miscellaneous compounds after activity-guided fractionation against MK-1, HeLa, and B16F10 cells [14]. Furthermore, three other phenylethanoids (21-23) isolated from the leaves of Ligustrum purpurascens (Oleaceae) were also evaluated [15]. It was remarkable that the effect of phenylethanoids (17-23) against B16F10 cells was more potent than their effects against HeLa and MK-1 cells. 
Table 1 Anti-proliferative activities of the plants extracts against MK-1, HeLa, B16F10, MT-1, and MT-2 cells

\begin{tabular}{|c|c|c|c|c|c|c|c|}
\hline Family & Scientific name & Parts & MK-1 & $\mathrm{HeLa}$ & B16F10 & MT-1 & MT-2 \\
\hline \multirow[t]{2}{*}{ Acanthaceae } & Dicliptera japonica & Aerial parts & - & - & + & - & - \\
\hline & Justicia procumbens & Whole part & - & + & + & $* * * *$ & $* *$ \\
\hline Actinidiaceae & Actinidia chinensis & Fruits & + & + & ++++ & - & - \\
\hline \multirow[t]{2}{*}{ Adoxaceae } & Sambucus chinensis & Leaves & - & - & - & - & - \\
\hline & & Stems & - & - & - & - & - \\
\hline Aizoaceae & Tetragonia expansa & Whole part & - & - & + & - & - \\
\hline \multirow[t]{7}{*}{ Amaranthaceae } & Achyranthes fauriei & Roots & +++ & ++++ & - & - & - \\
\hline & Celosia argentea & Seeds & - & - & - & - & - \\
\hline & Chenopodium ambrosidoides & Aerial parts & - & - & - & - & - \\
\hline & Chenopodium ambrosidoides var. anthelminticum & Leaves & + & - & + & - & - \\
\hline & & Stems & - & - & - & NT & NT \\
\hline & Chenopodium graveolens & Aerial parts & ++ & + & + & - & $* *$ \\
\hline & Gomphrena globosa & Whole part & - & - & - & - & - \\
\hline Amaryllidaceae & Allium sativum var. pekinense & Bulbs & +++ & + & ++++ & - & - \\
\hline \multirow[t]{5}{*}{ Anacardiaceae } & Mangifera indica & Barks & ++++++ & ++++++ & ++++++ & - & - \\
\hline & & Leaves & + & + & ++++ & $* *$ & - \\
\hline & & Peels & + & + & + & $* *$ & $* *$ \\
\hline & & Pulp & - & - & - & - & - \\
\hline & & Seeds & +++ & + & ++++ & $* *$ & $* *$ \\
\hline \multirow[t]{9}{*}{ Annonaceae } & Annona cherimola & Barks & + & - & + & $* * * *$ & $* * * *$ \\
\hline & & Leaves & ++++++ & ++++++ & ++++++ & $* *$ & $* *$ \\
\hline & Annona muricata & Leaves & +++ & + & ++++++ & - & - \\
\hline & & Stems & +++++ & + & ++++++ & $* *$ & - \\
\hline & Annona reticulata & Barks & + & - & - & - & - \\
\hline & & heartwoods & + & - & - & NT & NT \\
\hline & & Leaves & +++ & + & + & $* * * * * * * *$ & $* * * * * * * *$ \\
\hline & Annona squamosa & Leaves & +++++ & ++++++ & ++++++ & $* * * * * *$ & $* * * * * *$ \\
\hline & & Twigs & +++ & +++++ & +++++ & $* *$ & - \\
\hline \multirow[t]{20}{*}{ Apiaceae } & Angelica acutiloba & Fruits & + & - & - & NT & NT \\
\hline & & Leaves & - & - & - & NT & NT \\
\hline & & Roots & - & - & - & - & - \\
\hline & Angelica dahurica & Fruits & ++ & - & + & $* *$ & $* *$ \\
\hline & Angelica decursiva & Aerial parts & - & - & - & - & - \\
\hline & & Leaves & ++ & - & - & NT & NT \\
\hline & & Fruits & + & - & +++ & $* *$ & $* *$ \\
\hline & & Roots & + & - & - & $* *$ & $* *$ \\
\hline & Angelica japonica & Fruits & ++ & - & ++ & NT & NT \\
\hline & & Leaves & - & - & - & NT & NT \\
\hline & & Roots & +++ & - & - & NT & NT \\
\hline & Angelica keiskei & Aerial parts & - & - & + & - & - \\
\hline & & Leaves & - & - & - & NT & NT \\
\hline & & Fruits & - & - & + & - & - \\
\hline & & Roots & + & - & ++ & $* *$ & $* *$ \\
\hline & Angelica kiusiana & Leaves & - & - & + & - & - \\
\hline & Angelica pubescens & Roots & + & + & + & - & - \\
\hline & Anethum graveolens & Fruits & - & - & - & NT & NT \\
\hline & & Leaves & - & - & + & NT & NT \\
\hline & & Roots & +++ & - & - & NT & NT \\
\hline
\end{tabular}


Table 1 continued

\begin{tabular}{|c|c|c|c|c|c|c|c|}
\hline Family & Scientific name & Parts & MK-1 & $\mathrm{HeLa}$ & B16F10 & MT-1 & MT-2 \\
\hline & Anthriscus cerefolium & Fruits & ++ & - & - & NT & NT \\
\hline & \multirow[t]{3}{*}{ Anthriscus sylvestris } & Fruits & ++++++ & ++++++ & ++++++ & NT & NT \\
\hline & & Leaves & ++++++ & ++++++ & ++++++ & NT & NT \\
\hline & & Roots & ++++++ & ++++++ & ++++++ & NT & NT \\
\hline & Bupleurum falcatum & Leaves & - & - & - & NT & NT \\
\hline & Bupleurum rotundifolium & Fruits & +++++ & ++ & +++ & NT & NT \\
\hline & \multirow[t]{3}{*}{ Carum carvi } & Fruits & ++ & - & + & NT & NT \\
\hline & & Leaves & + & - & - & NT & NT \\
\hline & & Roots & ++++ & - & + & NT & NT \\
\hline & \multirow[t]{2}{*}{ Centella asiatica } & Leaves & ++ & - & ++ & NT & NT \\
\hline & & Aerial parts & + & - & +++ & - & - \\
\hline & Cnidium monnieri & Fruits & ++ & ++ & +++ & - & - \\
\hline & \multirow[t]{2}{*}{ Cnidium officinale } & Leaves & - & - & - & NT & NT \\
\hline & & Rhizomes & - & - & + & - & - \\
\hline & \multirow[t]{2}{*}{ Coriandrum sativum } & Leaves & - & - & - & - & - \\
\hline & & Fruits & ++ & - & ++ & NT & NT \\
\hline & Cryptotaenia japonica & Leaves & ++ & - & + & NT & NT \\
\hline & Foeniculum vulgare & Leaves & + & - & + & NT & NT \\
\hline & Glehnia littoralis & Fruits & +++++ & - & +++ & NT & NT \\
\hline & \multirow[t]{2}{*}{ Heracleum moellendorffii } & Leaves & + & - & - & NT & NT \\
\hline & & Roots & +++ & - & - & NT & NT \\
\hline & \multirow[t]{2}{*}{ Osmorhiza aristata } & Aerial parts & - & - & ++++++ & - & - \\
\hline & & Roots & - & - & - & $* *$ & $* *$ \\
\hline & \multirow[t]{3}{*}{ Peucedanum japonicum } & Leaves & - & - & - & - & - \\
\hline & & Stems, Root barks & + & + & - & $* *$ & $* *$ \\
\hline & & Woods & - & - & - & - & - \\
\hline & Peucedanum praeruptorum & Roots & - & - & - & $* *$ & $* *$ \\
\hline & \multirow[t]{3}{*}{ Torilis japonica } & Fruits & ++++ & - & ++++ & NT & NT \\
\hline & & Leaves & - & - & - & NT & NT \\
\hline & & Roots & +++ & - & + & NT & NT \\
\hline \multirow[t]{5}{*}{ Apocynaceae } & Apocynum venetum & Whole part & + & - & + & - & - \\
\hline & \multirow[t]{2}{*}{ Cerbera manghas } & Barks & NT & NT & NT & $* *$ & $* *$ \\
\hline & & Leaves & +++ & ++++ & - & $* * * *$ & $* * * *$ \\
\hline & Trachelospermum jasminoides & Aerial parts & - & - & - & $* *$ & $* *$ \\
\hline & Trachelospermum liukiuense & Aerial parts & - & - & + & - & $* *$ \\
\hline \multirow[t]{6}{*}{ Aquifoliaceae } & \multirow[t]{2}{*}{ Ilex cornuta } & Fruits & - & - & + & - & - \\
\hline & & Leaves & + & - & + & $* *$ & $* * * *$ \\
\hline & Ilex kudingcha & Leaves & NT & NT & NT & - & - \\
\hline & Ilex latifolia & Leaves & - & - & + & - & - \\
\hline & Ilex rotumda & Fruits & - & - & - & $* *$ & $* *$ \\
\hline & & Leaves & + & + & + & $* *$ & $* *$ \\
\hline \multirow[t]{2}{*}{ Araceae } & Arisaema ringens & Tubers & + & - & + & - & - \\
\hline & Pinellia ternata & Tubers & - & - & - & - & - \\
\hline \multirow[t]{7}{*}{ Araliaceae } & Aralia cordata & Roots & - & - & - & - & - \\
\hline & \multirow[t]{2}{*}{ Aralia elata } & Barks & + & - & ++++ & - & - \\
\hline & & Leaves & + & - & + & - & - \\
\hline & \multirow[t]{3}{*}{ Dendropanax trifidus } & Barks & - & - & + & - & - \\
\hline & & Fruits & - & - & + & - & - \\
\hline & & Leaves & + & + & +++ & - & - \\
\hline & Eleutherococcus senticosus & Root barks & - & - & + & $* *$ & $* *$ \\
\hline
\end{tabular}


Table 1 continued

\begin{tabular}{|c|c|c|c|c|c|c|c|}
\hline Family & Scientific name & Parts & MK-1 & $\mathrm{HeLa}$ & $\mathrm{B} 16 \mathrm{~F} 10$ & MT-1 & MT-2 \\
\hline & Fatsia japonica & Barks & + & - & - & - & - \\
\hline & & Leaves & + & - & - & - & - \\
\hline & & Roots & + & - & - & - & - \\
\hline & Hedera rhombea & Fruits & - & - & + & - & - \\
\hline & & Leaves & - & - & + & - & $* *$ \\
\hline & & Stems & - & - & + & $* *$ & $* *$ \\
\hline & Hydrocotyle nepalensis & Aerial parts & - & - & + & - & - \\
\hline & Schefflera arboricola & Leaves & + & + & + & - & - \\
\hline & Tetrapanax papyriferum & Leaves & +++ & ++++ & + & $* *$ & - \\
\hline & & Woods & + & + & + & - & - \\
\hline Araucariaceae & Araucaria heterophylla & Leaves & ++++ & +++++ & ++++++ & - & - \\
\hline \multirow[t]{4}{*}{ Aristolochiaceae } & Aristolochia spp. & Roots & + & + & - & $* * * *$ & $* * * *$ \\
\hline & Asarum nipponicum & Aerial parts & + & - & - & - & - \\
\hline & & Roots & + & - & - & $* * * *$ & $* * * *$ \\
\hline & Asarum sieboldii & Roots & + & - & + & - & - \\
\hline \multirow[t]{7}{*}{ Asclepiadaceae } & Asclepias curassavica & Leaves & + & ++++ & + & $* *$ & $* *$ \\
\hline & & Roots & +++ & ++++ & - & $* *$ & $* * * *$ \\
\hline & & Stems & +++ & ++++ & - & $* * * *$ & $* * * *$ \\
\hline & Cynanchum caudatum & Leaves & - & - & + & - & - \\
\hline & Marsdenia cundurango & Roots & - & - & - & - & - \\
\hline & Marsdenia tomentosa & Leaves & - & - & + & - & - \\
\hline & & Stems & - & - & - & - & - \\
\hline \multirow[t]{9}{*}{ Asclepiadaceae } & Metaplexis japonica & Aerial parts & - & - & - & NT & NT \\
\hline & & Roots & - & - & - & - & - \\
\hline & Periploca spp. & Root barks & ++++ & +++ & ++ & $* *$ & $* *$ \\
\hline & Tylophora ovata & Fresh leaves & ++++ & +++ & ++++ & $* * * *$ & $* * * *$ \\
\hline & & Twigs & +++++ & +++++ & +++++ & $* * * *$ & $* * * *$ \\
\hline & Tylophora ovata var. brownii & Fresh leaves & ++++++ & ++++++ & ++++++ & $* * * *$ & $* * * *$ \\
\hline & & Twigs & NT & NT & NT & - & - \\
\hline & Tylophora tanakae & Aerial parts & ++++++ & ++++++ & ++++++ & $* * * *$ & $* * * * * *$ \\
\hline & & Roots & ++++++ & ++++++ & ++++++ & $* * * * * *$ & $* * * * * *$ \\
\hline \multirow[t]{4}{*}{ Asparagaceae } & Anemarrhena asphodeloides & Roots, Rhizomes & - & - & - & $* *$ & $* *$ \\
\hline & Dracaena draco & Barks & - & - & +++ & - & - \\
\hline & & Leaves & - & - & ++++ & - & - \\
\hline & Ophiopogon japonicus & Tubers & - & - & - & - & - \\
\hline \multirow[t]{16}{*}{ Asteraceae } & Achillea millefolium & Leaves & - & - & + & - & - \\
\hline & & Stems & - & - & + & - & - \\
\hline & Adenocaulon himalaicum & Aerial parts & + & + & + & - & $* *$ \\
\hline & & Roots & - & - & - & - & - \\
\hline & Adenostemma lavenia & Aerial parts & + & - & + & - & $* *$ \\
\hline & Arctium lappa & Roots & - & - & - & - & - \\
\hline & & Seeds & + & - & ++ & - & - \\
\hline & Artemisia absinthium & Aerial parts & + & - & - & $* *$ & $* *$ \\
\hline & & Leaves & - & - & - & - & - \\
\hline & & Roots & + & - & + & $* *$ & $* *$ \\
\hline & & Stems & - & - & - & - & - \\
\hline & Artemisia campestris & Aerial parts & - & - & - & - & - \\
\hline & Artemisia capillaris & Aerial parts & + & - & + & - & $* *$ \\
\hline & & Roots & - & - & - & - & - \\
\hline & Artemisia ludoviciana var. mexicana & Aerial parts & +++ & + & +++ & - & - \\
\hline & Aster spathulifolius & Leaves & + & - & +++ & $* *$ & $* *$ \\
\hline
\end{tabular}


Table 1 continued

\begin{tabular}{|c|c|c|c|c|c|c|c|}
\hline Family & Scientific name & Parts & MK-1 & $\mathrm{HeLa}$ & B 16 F10 & MT-1 & MT-2 \\
\hline & & Stems & + & - & + & $* *$ & $* *$ \\
\hline & Aster verticillatum & Aerial parts & + & - & + & - & - \\
\hline & Bidens frondosa & Aerial parts & + & + & + & - & - \\
\hline & & Roots, Rhizomes & ++++ & ++++ & +++ & $* *$ & $* *$ \\
\hline & Carthamus tinctorius & Flowers & - & - & - & - & - \\
\hline & Centaurea benedictus & Leaves & + & - & + & NT & NT \\
\hline & Chrysanthemum vulgare & Aerial parts & + & + & + & $* *$ & $* *$ \\
\hline & Cichorium intybus & Aerial parts & + & - & + & - & - \\
\hline & & Roots & + & - & + & - & - \\
\hline & Cosmos bipinnatus & Seeds & - & - & - & - & - \\
\hline & Crassocephalum crepidioides & Aerial parts & - & - & + & - & - \\
\hline & & Roots, Rhizomes & - & - & + & - & - \\
\hline & Crepidiastrum lanceolatum & Aerial parts & - & - & - & - & - \\
\hline & & Roots & ++++ & ++++++ & ++++++ & - & - \\
\hline & Eclipta prostrata & Whole part & + & - & + & - & - \\
\hline & Eupatorium stoechadosmum & Leaves & - & - & - & - & - \\
\hline & & Roots & + & +++ & + & - & - \\
\hline & & Stems & - & - & - & - & - \\
\hline & Euryops pectinatus & Leaves & - & - & + & - & - \\
\hline & & Stems & - & - & - & - & - \\
\hline & Helianthus annuиs & Aerial parts & - & - & - & $* *$ & $* *$ \\
\hline & Inula helenium & Roots & +++ & +++ & +++ & NT & NT \\
\hline & Ligularia japonica & Leaves & - & - & - & $* *$ & - \\
\hline & & Roots & - & - & - & - & - \\
\hline & Neurolaena lobata & Leaves & - & + & + & NT & NT \\
\hline & Parasenecio tebakoensis & Aerial parts & + & - & + & - & - \\
\hline & Santolina chamaecyparissus & Leaves & - & - & + & - & - \\
\hline & & Stems & - & - & +++ & - & - \\
\hline & Saussurea lappa & Roots & +++++ & +++++ & +++++ & $* *$ & $* * * *$ \\
\hline & Senecio vulgaris & Whole part & - & - & - & - & - \\
\hline & Siegesbeckia glabrescens & Leaves & + & + & - & - & - \\
\hline & & Roots & - & - & - & - & - \\
\hline & Sonchus asper & Aerial parts & - & - & + & - & - \\
\hline & Tagetes patula & Aerial parts & - & - & + & - & $* *$ \\
\hline & & Roots & - & - & + & - & - \\
\hline & Tridax procumbens & Leaves & - & - & - & NT & NT \\
\hline & Tussilago farfara & Roots & + & - & + & - & - \\
\hline & Wedelia prostrata & Whole part & - & - & + & $* *$ & $* *$ \\
\hline & Xanthium strumarium & Fruits & - & - & - & - & - \\
\hline Balsaminaceae & Impatiens textori & Aerial parts & - & - & + & $* *$ & $* *$ \\
\hline \multirow[t]{7}{*}{ Berberidaceae } & Berberis japonica & Leaves & + & ++ & +++ & - & $* *$ \\
\hline & & Roots & + & + & ++++ & $* *$ & $* *$ \\
\hline & & Stems & + & + & ++++ & $* *$ & $* * * *$ \\
\hline & Epimedium grandiflorum subsp. & Roots, Rhizomes & - & - & - & - & - \\
\hline & Epimedium sagittatum & Aerial parts & +++++ & ++++ & ++++ & - & - \\
\hline & Nandina domestica & Barks & + & + & +++ & $* *$ & - \\
\hline & & Leaves & ++ & - & ++++ & - & - \\
\hline \multirow[t]{2}{*}{ Bignoniaceae } & Pseudocalymma alliaceum & Aerial parts & - & - & + & $* *$ & - \\
\hline & Tabebuia spp. & Barks & + & + & + & $* *$ & $* *$ \\
\hline Boraginaceae & Lithospermum officinale var. erythrorhizon & Roots & - & - & - & - & - \\
\hline Brassicaceae & Isatis indigotica & Fruits & - & - & + & $* *$ & $* *$ \\
\hline
\end{tabular}


Table 1 continued

\begin{tabular}{|c|c|c|c|c|c|c|c|}
\hline Family & Scientific name & Parts & MK-1 & $\mathrm{HeLa}$ & $\mathrm{B} 16 \mathrm{~F} 10$ & MT-1 & MT-2 \\
\hline & & Leaves & - & - & - & - & - \\
\hline & & Roots & - & - & - & - & - \\
\hline & Lepidium apetalum & Seeds & +++++ & ++++ & - & $* *$ & $* *$ \\
\hline & Lepidium virginicum & Whole part & - & - & + & - & - \\
\hline & Thlapsi arvense & Seeds & - & - & - & - & - \\
\hline \multirow[t]{3}{*}{ Burseraceae } & Bursera simaruba & Fruits & + & ++ & + & - & - \\
\hline & & Leaves & +++ & + & + & $* *$ & $* *$ \\
\hline & & Woods & + & ++ & + & NT & NT \\
\hline Campanulaceae & Codonopsis spp. & Roots & - & - & - & - & - \\
\hline Cannabaceae & Humulus japonicus & Aerial parts & - & - & + & $* *$ & $* *$ \\
\hline \multirow[t]{3}{*}{ Caprifoliaceae } & Lonicera japonica & Flowers & - & - & - & - & - \\
\hline & & Leaves & + & - & + & - & - \\
\hline & & Stems & - & - & + & - & - \\
\hline \multirow[t]{3}{*}{ Caricaceae } & Carica papaya & Barks & - & - & - & - & - \\
\hline & & Leaves & + & - & + & - & - \\
\hline & & Roots & - & - & - & - & - \\
\hline \multirow[t]{2}{*}{ Caryophyllaceae } & Agrostemma githago & Seeds & + & - & + & - & - \\
\hline & Vaccaria segetalis & Seeds & + & - & + & - & - \\
\hline \multirow[t]{6}{*}{ Celastraceae } & Celastrus orbiculatus & Vines & - & - & - & - & - \\
\hline & Euonymus alatus & Barks & - & - & +++ & NT & NT \\
\hline & Euonymus japonicus & Barks & +++ & + & ++++ & $* *$ & $* *$ \\
\hline & & Leaves & - & - & ++ & - & - \\
\hline & Maytenus diversifolia & Leaves & - & - & + & - & - \\
\hline & & Stems & +++ & +++ & +++ & NT & NT \\
\hline Chloranthaceae & Sarcandra glabra & Roots & - & +++ & + & - & - \\
\hline \multirow[t]{7}{*}{ Clusiaceae } & Garcinia subelliptica & Barks & + & + & ++++++ & - & - \\
\hline & & Heartwoods & - & - & - & - & - \\
\hline & & Leaves & + & - & + & - & - \\
\hline & Garcinia xanthochymus & Leaves & - & + & - & NT & NT \\
\hline & & Pulp & + & - & - & - & - \\
\hline & & Seeds & + & + & - & $* *$ & $* *$ \\
\hline & & Stems & + & + & + & NT & NT \\
\hline Combretaceae & Terminalia chebula & Fruits & + & +++ & ++++ & $* *$ & $* *$ \\
\hline Commelinaceae & Commelina communis & Whole part & - & - & + & - & - \\
\hline \multirow[t]{2}{*}{ Cornaceae } & Camptotheca acuminata & Fruits & +++++ & ++++ & ++++ & $* *$ & $* *$ \\
\hline & Cornus officinalis & Fruits & - & - & - & - & - \\
\hline \multirow[t]{6}{*}{ Crassulaceae } & Bryophyllum pinnatum & Aerial parts & + & - & + & - & - \\
\hline & & Roots & +++++ & +++++ & + & $* *$ & $* * * *$ \\
\hline & Hylotelephium erythrosticum & Roots & + & + & ++ & - & - \\
\hline & Orostachys japonicus & Whole part & - & - & + & - & - \\
\hline & Sedum aizoon var. floribundum & Roots & +++ & + & +++ & - & - \\
\hline & Sedum tomentosum & Whole part & - & - & + & - & - \\
\hline \multirow[t]{8}{*}{ Cucurbitaceae } & Actinostemma lobatum & Aerial parts & + & + & - & - & - \\
\hline & Citrullus colocynthis & Seeds & - & - & - & - & - \\
\hline & Gynostemma pentaphyllum & Aerial parts & - & - & - & - & - \\
\hline & Lagenaria leucantha var. gourda & Fruits & +++++ & ++++++ & + & $* * * *$ & $* *$ \\
\hline & & Leaves & - & - & + & - & - \\
\hline & & Roots & + & +++ & + & - & - \\
\hline & & Seeds & ++++ & +++++ & + & - & - \\
\hline & & Stems & +++ & +++ & + & - & - \\
\hline
\end{tabular}


Table 1 continued

\begin{tabular}{|c|c|c|c|c|c|c|c|}
\hline Family & Scientific name & Parts & MK-1 & $\mathrm{HeLa}$ & $\mathrm{B} 16 \mathrm{~F} 10$ & MT-1 & MT-2 \\
\hline & Lagenaria leucantha var. microcarpa & Fruits & +++++ & +++++ & +++ & NT & NT \\
\hline & & Seeds & ++++ & +++++ & +++ & - & - \\
\hline & Luffa acutangula & Aerial parts & + & + & + & ** & $* *$ \\
\hline & & Seeds & ++ & ++++++ & ++++++ & $* *$ & $* * * *$ \\
\hline & Luffa aegyptiaca & Fruits & - & - & - & - & - \\
\hline & Momordica charantia & Aerial parts & - & - & - & - & - \\
\hline & & Fruits & - & - & - & NT & NT \\
\hline & & Roots & - & - & - & - & - \\
\hline & Momordica cochinchinensis & Seeds & ++++++ & ++++++ & ++++++ & - & - \\
\hline & Sicana odorifera & Fruits & + & + & - & NT & NT \\
\hline & Trichosanthes kirilowii var. japonica & Roots & +++ & ++++ & + & - & - \\
\hline \multirow[t]{8}{*}{ Cupressaceae } & Biota orientalis & Leaves & ++ & +++ & ++++ & $* *$ & $* *$ \\
\hline & & Stems & + & + & + & - & - \\
\hline & Juniperus chinensis var. kaizuka Hort. & Leaves & + & ++++ & + & ** & $* *$ \\
\hline & & Stems & + & + & + & $* * * *$ & $* *$ \\
\hline & Juniperus rigida & Leaves & +++++ & ++++++ & ++++++ & $* * * *$ & $* * * *$ \\
\hline & & Stems & + & ++++ & ++++ & $* *$ & - \\
\hline & Thuja occidentalis & Leaves & ++++++ & ++++++ & ++++++ & $* * * * * *$ & $* * * * * *$ \\
\hline & & Stems & + & ++++ & +++ & $* * * * * *$ & $* *$ \\
\hline \multirow[t]{3}{*}{ Cycadaceae } & Cycas revoluta & Leaves & - & - & + & - & - \\
\hline & & Peels & - & - & + & - & - \\
\hline & & Seed kernels & - & - & - & - & - \\
\hline \multirow[t]{2}{*}{ Daphniphyllaceae } & Daphniphyllum macropodum & Barks & - & + & - & - & - \\
\hline & & Leaves & - & - & + & - & - \\
\hline \multirow[t]{2}{*}{ Elaeocarpaceae } & Elaeocarpus sylvestris var. ellipticus & Barks & +++ & +++ & ++++ & $* *$ & $* *$ \\
\hline & & Leaves & +++ & + & ++++ & - & $* *$ \\
\hline Eucommiaceae & Eucommia ulmoides & Barks & - & - & - & $* *$ & $* *$ \\
\hline \multirow[t]{9}{*}{ Euphorbiaceae } & Acalypha australis & Roots & ++ & + & + & - & - \\
\hline & Croton spp. & Leaves & + & - & + & - & - \\
\hline & Euphorbia helioscopia & Aerial parts & - & + & + & $* *$ & $* *$ \\
\hline & & Roots & - & - & + & - & $* *$ \\
\hline & Euphorbia jolkini & Aerial parts & + & ++ & ++++ & - & - \\
\hline & & Roots & + & ++ & ++++ & - & - \\
\hline & Euphorbia supina & Whole part & +++ & + & + & - & - \\
\hline & Euphorbia tirucalli & Aerial parts & ++ & + & + & - & - \\
\hline & Hura polyandra & Seeds & +++ & - & - & - & - \\
\hline \multirow[t]{15}{*}{ Fabaceae } & Acacia melanoxylon & Barks & +++ & + & +++ & - & - \\
\hline & & Leaves & + & + & + & - & $* *$ \\
\hline & Apios americana & Flowers & NT & NT & NT & - & - \\
\hline & Astragalus membranaceus & Roots & - & + & - & - & - \\
\hline & Canavalia gladiata & Roots & - & + & - & - & $* *$ \\
\hline & & Seeds & - & - & + & $* *$ & $* *$ \\
\hline & Cassia obtusifolia & Seeds & - & + & + & - & - \\
\hline & Crotalaria juncea & Leaves & + & - & + & - & $* *$ \\
\hline & & Seeds & - & - & - & - & - \\
\hline & & Stems & - & - & + & - & - \\
\hline & Erythrina variegata var. orientalis & Barks & - & + & - & - & - \\
\hline & Euchresta japonica & Roots & - & + & - & - & $* *$ \\
\hline & Eysenhardtia polystachia & Woods & + & + & +++ & - & - \\
\hline & Gliricidia sepium & Leaves & + & - & ++ & - & - \\
\hline & Glycyrrhiza pallidiflora & Underground parts & - & + & - & - & - \\
\hline
\end{tabular}


Table 1 continued

\begin{tabular}{|c|c|c|c|c|c|c|c|}
\hline Family & Scientific name & Parts & MK-1 & HeLa & B16F10 & MT-1 & MT-2 \\
\hline & Glycyrrhiza uralensis & Roots & - & + & - & - & - \\
\hline & Haematoxylum brasileto & Woods & ++++ & +++ & ++++ & $* * * *$ & $* * * *$ \\
\hline & Lonchocarpus oxacensis & Roots & + & - & - & NT & NT \\
\hline & Lonchocarpus unifoliolatus & Roots & + & - & - & NT & NT \\
\hline & Medicago polymorpha & Whole part & - & - & - & - & - \\
\hline & Melilotus officinalis & Whole part & - & + & - & - & - \\
\hline & Psoralea corylifolia & Seeds & ++ & ++ & ++ & - & $* *$ \\
\hline & Rhynchosia volubilis & Seeds & +++ & ++ & ++++ & $* *$ & - \\
\hline & Sophora japonica & Fruits & - & - & + & $* *$ & $* *$ \\
\hline & Trifolium dubium & Aerial parts & - & + & - & - & - \\
\hline & Zornia spp. & Leaves & + & - & + & - & $* *$ \\
\hline \multirow[t]{2}{*}{ Gelsemiaceae } & Gelsemium sempervirens & Leaves & - & - & +++ & - & - \\
\hline & & Stems & - & - & + & - & - \\
\hline \multirow[t]{2}{*}{ Geraniaceae } & Pelargonium graveolens & Leaves & +++ & + & ++++ & - & ** \\
\hline & & Stems & +++ & + & ++++ & - & $* *$ \\
\hline Iridaceae & Crocosmia aurea & Bulbs & - & - & - & - & - \\
\hline Jugulandaceae & Juglans mandshurica var. sachalinensis & Barks & ++ & ++ & +++ & - & - \\
\hline \multirow[t]{28}{*}{ Lamiaceae } & Ajuga decumbens & Whole part & - & - & - & - & - \\
\hline & Ajuga reptans & Leaves & - & - & - & - & - \\
\hline & & Roots & - & - & - & - & - \\
\hline & Caryopteris incana & Aerial parts & - & - & + & - & - \\
\hline & Clerodendron thomsonaiae & Leaves & - & - & - & NT & NT \\
\hline & Clerodendrum bungei & Flowers & - & - & - & - & - \\
\hline & & Leaves & - & - & ++ & - & - \\
\hline & & Stems & - & - & - & - & - \\
\hline & Clerodendrum trichotomum & Barks & + & - & +++ & - & - \\
\hline & & Flowers & - & - & - & - & - \\
\hline & & Fruits & - & - & + & - & - \\
\hline & & Leaves & + & - & +++ & - & - \\
\hline & Elsholtzia ciliata & Aerial parts & + & - & + & - & - \\
\hline & Glechoma longituba & Whole part & - & - & - & - & - \\
\hline & Isodon japonicus & Leaves & + & + & ++++ & - & - \\
\hline & & Roots & + & + & + & - & - \\
\hline & & Stems & + & + & ++++ & - & - \\
\hline & Lamium amplexicaule & Whole part & - & - & - & - & - \\
\hline & Leonurus sibiricus & Aerial parts & - & - & - & $* *$ & $* *$ \\
\hline & & Roots & - & - & + & - & - \\
\hline & & Seeds & - & - & + & - & - \\
\hline & Rosmarinus officinalis & Leaves & +++ & + & ++++ & $* *$ & $* *$ \\
\hline & Salvia miltiorrhiza & Roots & + & - & + & - & - \\
\hline & Scutellaria baicalensis & Roots & + & + & + & - & - \\
\hline & Scutellaria barbata & Whole part & - & - & + & $* *$ & $* *$ \\
\hline & Teucrium japonicum & Leaves & - & - & +++ & - & - \\
\hline & Vitex trifolia & Branches & NT & NT & NT & - & - \\
\hline & & Leaves & NT & NT & NT & - & - \\
\hline \multirow[t]{5}{*}{ Lauraceae } & Cinnamomum cassia & Barks & + & + & + & $* *$ & $* *$ \\
\hline & Lindera strychnifolia & Roots & + & + & + & - & - \\
\hline & Persea americana & Leaves & +++++ & ++++++ & ++++++ & - & - \\
\hline & & Pulp & + & +++ & + & - & - \\
\hline & & Seeds & + & + & + & - & - \\
\hline
\end{tabular}


Table 1 continued

\begin{tabular}{|c|c|c|c|c|c|c|c|}
\hline Family & Scientific name & Parts & MK-1 & $\mathrm{HeLa}$ & B16F10 & MT-1 & MT-2 \\
\hline & & Twigs & + & + & + & - & - \\
\hline Liliaceae & Fritillaria verticillata var. thungergii & Bulbs & - & - & + & - & - \\
\hline \multirow[t]{3}{*}{ Lythraceae } & Cuphea hyssopifolia & Aerial parts & + & ++ & + & - & - \\
\hline & & Roots & ++ & + & + & - & - \\
\hline & Punica granatum & Peels & +++ & + & ++++ & $* *$ & $* *$ \\
\hline Magnoliaceae & Magnolia ovata & Barks & + & + & + & - & - \\
\hline \multirow[t]{18}{*}{ Malvaceae } & Abelmoschus manihot & Leaves & - & + & + & - & - \\
\hline & Althaea cannabina & Leaves & - & - & - & - & - \\
\hline & Chorisia speciosa & Immatured fruits & - & - & - & - & - \\
\hline & Corchoropsis tomentosa & Fruits & - & - & + & $* *$ & $* *$ \\
\hline & & Leaves & + & + & +++ & - & - \\
\hline & & Stems & - & - & + & - & - \\
\hline & Gossypium arboreum & Leaves & + & + & ++ & - & - \\
\hline & & Roots & + & + & ++ & - & - \\
\hline & & Stems & + & - & + & - & - \\
\hline & Gossypium brasiliensis & Leaves & ++ & ++ & ++ & - & - \\
\hline & & Roots & - & + & + & - & - \\
\hline & & Stems & - & + & + & - & - \\
\hline & Malvaviscus arboreus & Leaves & + & - & - & - & - \\
\hline & Pachira macrocarpa & Barks & + & + & + & $* *$ & $* *$ \\
\hline & & Leaves & + & - & + & - & $* *$ \\
\hline & Sterculia nobilis & Barks & - & - & - & - & - \\
\hline & & Heartwoods & - & - & - & - & - \\
\hline & & Leaves & ++ & + & + & $* *$ & $* *$ \\
\hline Meliaceae & Melia azedarach var. toosendan & Fruits & - & - & ++++ & $* *$ & $* *$ \\
\hline \multirow[t]{5}{*}{ Menispermaceae } & Cocculs trilobus & Fruits & - & - & + & - & - \\
\hline & & Leaves & + & + & + & - & - \\
\hline & & Vines & - & - & + & - & - \\
\hline & Stephania tetrandra & Roots & - & - & + & $* *$ & ** \\
\hline & Tinospora tuberculata & Stems & - & - & - & - & - \\
\hline \multirow[t]{5}{*}{ Moraceae } & Ficus carica & Leaves & - & - & + & - & - \\
\hline & Ficus pumila & Fruits & + & - & + & - & - \\
\hline & & Leaves & + & + & +++ & - & - \\
\hline & & Stems & - & - & + & - & - \\
\hline & Morus alba & Root barks & - & - & - & - & - \\
\hline \multirow[t]{2}{*}{ Myristicaceae } & Myristica fragrans & $\mathrm{MeOH}-\mathrm{oil}$ & ++ & ++ & ++ & NT & NT \\
\hline & & $\mathrm{MeOH}-\mathrm{ppt}$ & - & - & + & - & - \\
\hline \multirow[t]{2}{*}{ Muntingiaceae } & Muntingia calabura & Fruits & + & - & + & NT & NT \\
\hline & & Leaves & +++ & + & ++ & - & - \\
\hline \multirow[t]{11}{*}{ Myrtaceae } & Eugenia javanica & Barks & +++ & +++ & ++++ & - & - \\
\hline & & Leaves & + & + & +++ & - & - \\
\hline & Eugenia uniflora & Leaves & + & + & +++ & - & - \\
\hline & & Twigs & +++ & + & +++ & - & $* *$ \\
\hline & Psidium cattleyanum & Branches & +++ & + & + & $* *$ & - \\
\hline & & Fruits & - & - & - & - & - \\
\hline & & Leaves & + & + & + & - & - \\
\hline & Psidium guajava & Branches & + & + & +++ & - & - \\
\hline & & Leaves & + & + & + & - & - \\
\hline & Psidium littorale & Leaves & + & + & +++ & - & - \\
\hline & & Twigs & +++ & +++ & ++++ & - & $* *$ \\
\hline
\end{tabular}


Table 1 continued

\begin{tabular}{|c|c|c|c|c|c|c|c|}
\hline Family & Scientific name & Parts & MK-1 & HeLa & $\mathrm{B} 16 \mathrm{~F} 10$ & MT-1 & MT-2 \\
\hline \multirow[t]{2}{*}{ Nyctaginaceae } & \multirow[t]{2}{*}{ Mirabilis jalapa } & Leaves & - & - & + & - & $* *$ \\
\hline & & Roots & + & + & + & - & - \\
\hline \multirow[t]{7}{*}{ Oleaceae } & \multirow[t]{2}{*}{ Ligustrum japonicum } & Immatured fruits & + & - & + & - & - \\
\hline & & Leaves & + & + & +++ & NT & NT \\
\hline & \multirow[t]{2}{*}{ Ligustrum lucidum } & Fruits & + & - & + & - & - \\
\hline & & Leaves & - & - & ++ & - & - \\
\hline & Ligustrum ovalifolium & Leaves & - & - & + & - & - \\
\hline & Ligustrum purpurascens & Leaves & + & + & + & - & - \\
\hline & Ligustrum salicinum & Leaves & + & - & +++ & - & - \\
\hline Orchidaceae & Dendrobium spp. & Aerial parts & + & + & + & - & - \\
\hline Orobanchaceae & Cistanche deserticola & Stems & - & - & + & - & - \\
\hline \multirow[t]{2}{*}{ Oxalidaceae } & \multirow[t]{2}{*}{ Averrhoa carambola } & Barks & - & - & + & - & - \\
\hline & & Leaves & - & - & + & - & - \\
\hline Paeoniaceae & Paeonia lactiflora & Roots & - & - & - & - & - \\
\hline \multirow[t]{5}{*}{ Papaveraceae } & \multirow[t]{2}{*}{ Corydalis heterocarpa var. japonica } & Aerial parts & - & - & + & $* *$ & $* *$ \\
\hline & & Roots & + & - & - & - & - \\
\hline & Corydalis turtschaninovii forma yanhusuo & Tubers & + & + & ++ & - & $* *$ \\
\hline & \multirow[t]{2}{*}{ Macleaya cordata } & Aerial parts & +++ & +++ & +++ & - & - \\
\hline & & Underground parts & + & + & + & $* *$ & $* *$ \\
\hline \multirow[t]{2}{*}{ Phrymaceae } & \multirow[t]{2}{*}{ Phryma leptostachya } & Aerial parts & + & - & + & - & - \\
\hline & & Roots & + & + & + & - & - \\
\hline \multirow[t]{3}{*}{ Phyllanthaceae } & \multirow[t]{2}{*}{ Phyllanthus aciduse } & Leafstalks, Twigs & - & - & - & - & - \\
\hline & & Leaves & + & - & - & - & - \\
\hline & Phyllanthus urinaria & Whole parts & + & + & ++++ & - & - \\
\hline \multirow[t]{3}{*}{ Phytolaccaceae } & Petiveria alliacea & Leaves & + & + & +++ & NT & NT \\
\hline & Phytolacca americana & Roots & - & - & - & - & - \\
\hline & Rivina humilis & Aerial parts & - & - & + & - & - \\
\hline Piperaceae & Piper spp. & Leaves & + & - & + & $* *$ & $* *$ \\
\hline \multirow[t]{4}{*}{ Pittosporaceae } & \multirow[t]{4}{*}{ Pittosporum tobira } & Barks & - & - & - & - & - \\
\hline & & Fruits & + & + & + & - & - \\
\hline & & Leaves & + & - & + & - & - \\
\hline & & Peels & + & + & + & $* *$ & $* *$ \\
\hline \multirow[t]{5}{*}{ Plantaginaceae } & \multirow[t]{3}{*}{ Pentstemon gloxinioides } & Leaves & - & - & + & - & - \\
\hline & & Rhizomes & - & - & +++ & - & - \\
\hline & & Stems & - & - & + & - & - \\
\hline & Picrorhiza scrophulariiflora & Rhizomes & - & - & + & - & - \\
\hline & Russelia equisetiformis & Aerial parts & + & - & ++++ & - & - \\
\hline Plumbaginaceae & Plumbago capensis & Whole part & + & + & + & - & - \\
\hline Podocarpaceae & Podocarpus macrophyllus & Leaves & + & + & +++ & - & - \\
\hline & & Stems & + & + & ++++ & - & - \\
\hline Polygalaceae & Polygala tenuifolia & Roots & - & - & - & - & - \\
\hline Polygonaceae & Fallopia japonica & Roots & + & + & + & - & - \\
\hline & Polygonum orientale & Seeds & + & + & +++ & - & - \\
\hline & Polygonum tinctorium & Whole part & - & - & - & $* *$ & $* *$ \\
\hline & Rheum palmatum & Rhizomes & +++ & + & ++++ & - & - \\
\hline & Rumex acetosa & Roots, Rhizomes & - & - & + & $* *$ & $* *$ \\
\hline & Rumex japonicus & Roots, Rhizomes & - & - & ++ & - & $* *$ \\
\hline Polypodiaceae & Drynaria fortunei & Rhizomes & - & - & + & - & - \\
\hline & Phleboidum aureum & Dried roots & + & - & + & - & - \\
\hline & & Fresh roots & + & - & - & - & $* *$ \\
\hline & & Whole part & + & - & + & - & - \\
\hline Portulacaceae & Portulaca oleracea & Whole part & + & - & + & - & $* *$ \\
\hline
\end{tabular}


Table 1 continued

\begin{tabular}{|c|c|c|c|c|c|c|c|}
\hline Family & Scientific name & Parts & MK-1 & $\mathrm{HeLa}$ & $\mathrm{B} 16 \mathrm{~F} 10$ & MT-1 & MT-2 \\
\hline \multirow[t]{6}{*}{ Primulaceae } & \multirow[t]{3}{*}{ Ardisia crenata } & Leaves & - & - & +++ & - & - \\
\hline & & Roots & ++++ & + & +++ & $* * * *$ & $* * * *$ \\
\hline & & Stems & - & - & +++ & - & - \\
\hline & \multirow[t]{2}{*}{ Ardisia japonica } & Leaves & + & + & + & - & - \\
\hline & & Stems, Undergorund parts & + & + & +++ & - & - \\
\hline & Lysimachia japonbica & Whole part & - & - & + & - & - \\
\hline \multirow[t]{2}{*}{ Proteaceae } & \multirow[t]{2}{*}{ Macadamia ternifolia } & Leaves & + & + & +++ & - & - \\
\hline & & Twigs & +++ & ++ & +++ & NT & NT \\
\hline \multirow[t]{2}{*}{ Pteridaceae } & \multirow[t]{2}{*}{ Pteris multifida } & Aerial parts & - & - & - & - & - \\
\hline & & Roots, Rhizomes & + & + & + & - & - \\
\hline \multirow[t]{6}{*}{ Ranunculaceae } & \multirow[t]{2}{*}{ Cimicifuga simplex var. ramosa } & Aerial parts & - & - & + & - & - \\
\hline & & Underground parts & - & - & + & - & - \\
\hline & Clematis paniculata & Aerial parts & + & - & + & - & - \\
\hline & Coptis japonica & Rhizomes & +++++ & +++++ & ++++++ & $* *$ & $* *$ \\
\hline & \multirow[t]{2}{*}{ Thalictrum thunbergii } & Aerial parts & - & - & + & - & - \\
\hline & & Underground parts & - & - & +++ & - & - \\
\hline \multirow[t]{5}{*}{ Rhamnaceae } & \multirow[t]{2}{*}{ Berchemia racemosa } & Leaves & - & - & + & - & - \\
\hline & & Stems & - & - & + & - & - \\
\hline & Hovenia dulcis & Fruits & - & - & + & - & - \\
\hline & Zizyphus jujube var. jujuba & Fruits & - & - & - & - & - \\
\hline & Zizyphus jujube var. spinosa & Seeds & ++ & - & + & - & - \\
\hline Rehmanniaceae & Rehmannia glutinosa var. purpurea & Roots & - & - & - & - & - \\
\hline \multirow[t]{17}{*}{ Rosaceae } & Agrimonia pilosa & Whole part & - & - & ++ & - & - \\
\hline & Chaenomeles sinensis & Fruits & + & + & +++ & - & - \\
\hline & Crataegus cuneata & Fruits & - & + & - & - & - \\
\hline & \multirow[t]{3}{*}{ Eryobotrya japonica } & Barks & + & + & + & - & - \\
\hline & & Leaves & - & - & + & - & - \\
\hline & & Seeds & - & + & - & - & - \\
\hline & \multirow[t]{2}{*}{ Geum japonicum } & Aerial parts & + & + & + & - & - \\
\hline & & Roots & + & + & +++ & - & - \\
\hline & Potentilla chrysantha & Whole part & - & - & + & - & - \\
\hline & \multirow[t]{2}{*}{ Potentilla fragarioides var. major } & Aerial parts & - & - & + & - & - \\
\hline & & Underground parts & + & + & +++ & $* *$ & $* *$ \\
\hline & Potentilla indica & Whole part & + & - & + & - & - \\
\hline & Prunus armeniaca & Seeds kernels & - & + & - & - & - \\
\hline & Rosa multiflora & Fruits & - & - & + & - & - \\
\hline & \multirow[t]{2}{*}{ Rubus hirsutus } & Aerial parts & - & - & + & - & - \\
\hline & & Roots & + & - & + & - & - \\
\hline & Sanguisorba officinalis var. carnea & Roots & ++ & ++ & ++++ & - & - \\
\hline \multirow[t]{11}{*}{ Rubiaceae } & \multirow[t]{3}{*}{ Damnacanthus macrophyllus var. macrophyllus } & Leaves & - & ++ & ++ & - & - \\
\hline & & Roots & - & - & - & - & - \\
\hline & & Stems & - & - & - & - & - \\
\hline & Galium pogonanthum & Aerial part & - & - & + & - & $* *$ \\
\hline & Hamelia patens & Leaves, Twigs & + & - & ++ & - & - \\
\hline & Hedyotis diffusa & Whole part & - & - & - & - & - \\
\hline & Paederia scandens & Fruits & + & + & - & - & - \\
\hline & & Leaves & - & - & - & - & - \\
\hline & & Stems & - & - & - & - & - \\
\hline & Rubia argyi & Roots & ++++ & ++++ & ++++ & $* *$ & $* *$ \\
\hline & Uncaria rhynchophylla & Hooks & - & - & ++ & - & - \\
\hline Rutaceae & Boenninghausenia japonica & Aerial parts & + & +++ & + & $* *$ & $* *$ \\
\hline & & Roots & +++ & ++++ & + & $* * * * * *$ & $* *$ \\
\hline
\end{tabular}


Table 1 continued

\begin{tabular}{|c|c|c|c|c|c|c|c|}
\hline Family & Scientific name & Parts & MK-1 & $\mathrm{HeLa}$ & $\mathrm{B} 16 \mathrm{~F} 10$ & MT-1 & MT-2 \\
\hline & Citrus grandis & Peels & - & - & + & - & - \\
\hline & Citrus natsudaidai & Immatured fruits & - & - & - & - & - \\
\hline & Evodia rutaecarpa & Barks & - & - & + & - & - \\
\hline & & Fruits & +++ & +++ & ++++ & - & - \\
\hline & Orixa japonica & Leaves & + & + & + & - & - \\
\hline & & Stems & - & - & + & - & - \\
\hline & Phellodendron amurense & Barks & + & ++ & +++++ & $* *$ & $* * * *$ \\
\hline & Ruta graveolens & Aerial parts & - & + & ++++ & $* *$ & $* *$ \\
\hline & & Leaves & + & + & + & $* *$ & $* * * *$ \\
\hline & & Roots & ++++ & +++++ & +++ & $* *$ & $* * * *$ \\
\hline & Zanthoxylum ailanthoides & Barks & + & + & +++ & $* *$ & $* *$ \\
\hline & & Fruits & - & - & + & $* *$ & - \\
\hline & & Leaves & - & - & + & $* *$ & $* *$ \\
\hline & & Woods & - & - & + & - & $* *$ \\
\hline & Zanthoxylum bungeanum & Peels & - & - & + & $* *$ & $* *$ \\
\hline \multirow[t]{8}{*}{ Sapindaceae } & Aesculus turbinata & Seeds & ++ & - & - & - & - \\
\hline & Cardiospermum halicacabum & Seeds & - & - & + & - & - \\
\hline & Dimocarpus longan & Leaves & +++ & + & +++ & - & - \\
\hline & & Twigs & +++ & + & +++ & - & - \\
\hline & Litchi chinensis & Leaves & + & + & +++ & - & - \\
\hline & & Twigs & +++ & + & + & - & - \\
\hline & Sapindus mukurossi & Peels & +++ & +++ & +++ & $* * * *$ & $* *$ \\
\hline & & Seeds & - & - & + & - & - \\
\hline \multirow[t]{2}{*}{ Sapotaceae } & Chrysophyllum cainito & Leaves & + & + & + & NT & NT \\
\hline & Pouteria sapota & Seeds & + & - & - & - & - \\
\hline \multirow[t]{3}{*}{ Schisandraceae } & Kadsura japonica & Leaves & - & - & + & - & - \\
\hline & & Vines & + & - & + & - & $* *$ \\
\hline & Schisandra chinensis & Fruits & - & - & - & - & - \\
\hline \multirow[t]{2}{*}{ Scrophlariaceae } & Scrophularia buergeriana & Roots & - & - & - & - & - \\
\hline & Verbascum thapsus & Leaves & - & - & + & - & - \\
\hline Simaroubaceae & Picrasma quassioides & Woods & + & + & + & - & - \\
\hline \multirow[t]{2}{*}{ Smilacaceae } & Smilax china & Rhizomes & + & + & +++ & - & - \\
\hline & Smilax medica & Rhizomes & + & + & + & - & - \\
\hline \multirow[t]{14}{*}{ Solanaceae } & Brunfelsia latifolia & Leaves & + & - & + & - & - \\
\hline & Nicandra physalodes & Fruits & - & - & - & - & - \\
\hline & & Leaves & + & - & +++ & - & - \\
\hline & & Roots & +++ & - & +++ & - & - \\
\hline & & Stems & - & - & - & - & - \\
\hline & Physalis angulata & Aerial parts & ++++ & +++ & +++ & - & - \\
\hline & & Roots & - & - & - & - & - \\
\hline & Physalis pruinosa & Aerial parts & ++++++ & +++ & +++ & $* *$ & $* *$ \\
\hline & & Roots & +++++ & + & +++++ & $* * * *$ & $* * * *$ \\
\hline & Solanum mammosum & Aerial parts & + & - & + & - & - \\
\hline & & Roots & + & - & - & - & - \\
\hline & Solanum nigrum & Aerial parts & - & - & + & $* *$ & - \\
\hline & & Fruits & + & + & + & $* *$ & - \\
\hline & & Roots & + & + & +++ & $* *$ & $* *$ \\
\hline \multirow[t]{3}{*}{ Taxaceae } & Cephalotaxus harringtonia & Leaves & ++++ & ++++ & ++++ & $* * * *$ & $* * * *$ \\
\hline & & Stems, Twigs & +++++ & ++++ & +++++ & - & - \\
\hline & Torreya grandis & Seeds & ++ & + & +++ & - & - \\
\hline Theaceae & Camellia sinensis & Leaves & ++ & NT & NT & NT & NT \\
\hline Thymelaeaceae & Daphne genkwa & Flowers & + & + & + & $* *$ & $* *$ \\
\hline
\end{tabular}


Table 1 continued

\begin{tabular}{|c|c|c|c|c|c|c|c|}
\hline Family & Scientific name & Parts & MK-1 & $\mathrm{HeLa}$ & B16F10 & MT-1 & MT-2 \\
\hline & Daphne odora & Roots & - & - & ++++ & - & - \\
\hline & Edgeworthia chrysantha & Roots & - & - & + & - & - \\
\hline \multirow[t]{4}{*}{ Urticaceae } & Cecropia obtusifolia & Fresh leaves & + & - & + & - & - \\
\hline & & Leaves & ++ & + & + & - & - \\
\hline & Urtica dioica & Leaves, Twigs & - & - & - & - & - \\
\hline & Urtica thunbergiana & Aerial parts & - & - & + & - & - \\
\hline \multirow[t]{12}{*}{ Verbenaceae } & Aloysia triphylla & Leaves & +++ & + & + & - & - \\
\hline & Lantana camara var. aculeata & Leaves & +++ & +++ & + & - & - \\
\hline & & Stems & +++ & +++ & + & - & - \\
\hline & Lantana montevidensis & Leaves & ++++ & +++++ & +++++ & - & - \\
\hline & Lippia canescens & Aerial parts & - & - & + & - & - \\
\hline & Lippia dulcis & Aerial parts & + & - & ++++ & - & - \\
\hline & Lippia triphylla & Leaves & + & - & +++ & - & - \\
\hline & & Stems & - & - & + & - & - \\
\hline & Verbena brasiliensis & Aerial parts & + & - & + & - & - \\
\hline & & Roots & + & - & ++ & - & - \\
\hline & Verbena officinalis & Aerial parts & + & - & + & - & - \\
\hline & & Roots & - & - & - & - & - \\
\hline Vitaceae & Cayratia japonica & Aerial parts & - & - & + & - & - \\
\hline Xanthorrhoeaceae & Aloe ferox & Leaves & + & - & - & - & - \\
\hline \multirow[t]{5}{*}{ Zingiberaceae } & Alpinia japonica & Fruits & - & - & - & - & - \\
\hline & & Seeds & - & + & + & - & $* *$ \\
\hline & Curcuma zedoaria & Rhizomes & + & - & + & $* *$ & $* *$ \\
\hline & Hedychium coronarium & Rhizomes & + & + & + & $* *$ & $* *$ \\
\hline & Zingiber officinale & Rhizomes & + & + & ++ & $* *$ & $* * * *$ \\
\hline
\end{tabular}

The extracts are classified in the Angiosperm Phylogeny Group III system. $\mathrm{EC}_{50}$ values against MK-1, HeLa, and B16F10 cells $(<3.13 \mu \mathrm{g} / \mathrm{mL}$, $++++++; 3.13-6.25 \mu \mathrm{g} / \mathrm{mL},+++++; 6.25-12.5 \mu \mathrm{g} / \mathrm{mL},++++; 12.5-25 \mu \mathrm{g} / \mathrm{mL},+++; 25-50 \mu \mathrm{g} / \mathrm{mL},++; 50-100 \mu \mathrm{g} / \mathrm{mL},+;>100 \mu \mathrm{g} /$ $\mathrm{mL},-) . \mathrm{EC}_{50}$ values against MT-1 and MT-2 cells $(<0.1 \mu \mathrm{g} / \mathrm{mL}$, ********; $0.1-1 \mu \mathrm{g} / \mathrm{mL}, * * * * * * ; 1-10 \mu \mathrm{g} / \mathrm{mL}, * * * * ; 10-100 \mu \mathrm{g} / \mathrm{mL}, * * ;>100$ $\mu \mathrm{g} / \mathrm{mL},-)$

$N T$ not tested

Because the effects of compound $\mathbf{2 3}$ were extremely weak, this suggested the 3,4-dihydroxyphenethyl group is essential for the observed strong anti-proliferative activity. Furthermore, 3,4-dihydroxyphenethyl alcohol itself showed potent activity [13]. It is also known that treatment of phenylethanoids resulted in apoptotic cell death [16].

\section{Polyphenols (Fig. 4)}

Epidemiological studies have suggested that the consumption of green tea [Camellia sinensis (Theaceae)] provides protection against stomach cancer. In a rural area of northern Kyushu, Japan, a decreased risk of stomach cancer was also noted among people reporting a high consumption of green tea [17]. Fractionation of green tea extract, guided by the anti-proliferative activity against MK-1 cells, resulted in the isolation of six flavan-3-ols (24-29) together with the inactive glycosides of kaempferol and quercetin [18]. A study of their structure-activity relationships suggested that the presence of the three adjacent hydroxyl groups (pyrogallol or galloyl group) in the molecule is a key factor for enhancing the compound's activity. Six active polyphenols (30-35) were isolated from the seeds of Rhynchosia volubilis (Fabaceae) after activityguided fractionation against MK-1, HeLa, and B16F10 cells [19]. These compounds all showed much stronger inhibition against B16F10 cell growth than against HeLa and MK-1 cell growth. Gallic acid (31) with a free carboxyl group showed higher activity than its methyl ester (32). A hydrolysable tannin (36) and two condensed tannins $(37,38)$ isolated from Phyllanthus emblica (Phyllanthaceae) also showed potent activity [20] against three cell lines. It was proposed that the anti-cancer properties of polyphenols may be related to their ability to participate in a copper-dependent prooxidant mechanism [21].

\section{Flavones (Fig. 5)}

After activity-guided fractionation against MK-1, HeLa, and B16F10 cells, 11 active flavones $(\mathbf{3 9}-\mathbf{4 9})$ were isolated 
Table 2 Summary of the sensitivity of the plants extracts against MK-1, HeLa, B16F10, MT-1, and MT-2 cells

\begin{tabular}{llllll}
\hline Confirmed EC 50 activities & MK-1 $(\%)$ & HeLa $(\%)$ & B16F10 (\%) & MT-1 (\%) & MT-2 (\%) \\
\hline$<100 \mu \mathrm{g} / \mathrm{mL}$ & 55 & 39 & 70 & 23 & 28 \\
$<12.5 \mu \mathrm{g} / \mathrm{mL}$ & 7.6 & 7.8 & 12 & - & - \\
$<10 \mu \mathrm{g} / \mathrm{mL}$ & - & - & - & 4.5 & 5.3 \\
$<3.13 \mu \mathrm{g} / \mathrm{mL}$ & 1.9 & 2.8 & 3.6 & - & - \\
$<1 \mu \mathrm{g} / \mathrm{mL}$ & - & - & - & 1.1 & 0.9 \\
\hline
\end{tabular}

The percentages for each cell type represent the percent of the extracts with $\mathrm{EC}_{50} \mathrm{~s}$ within each category of activity
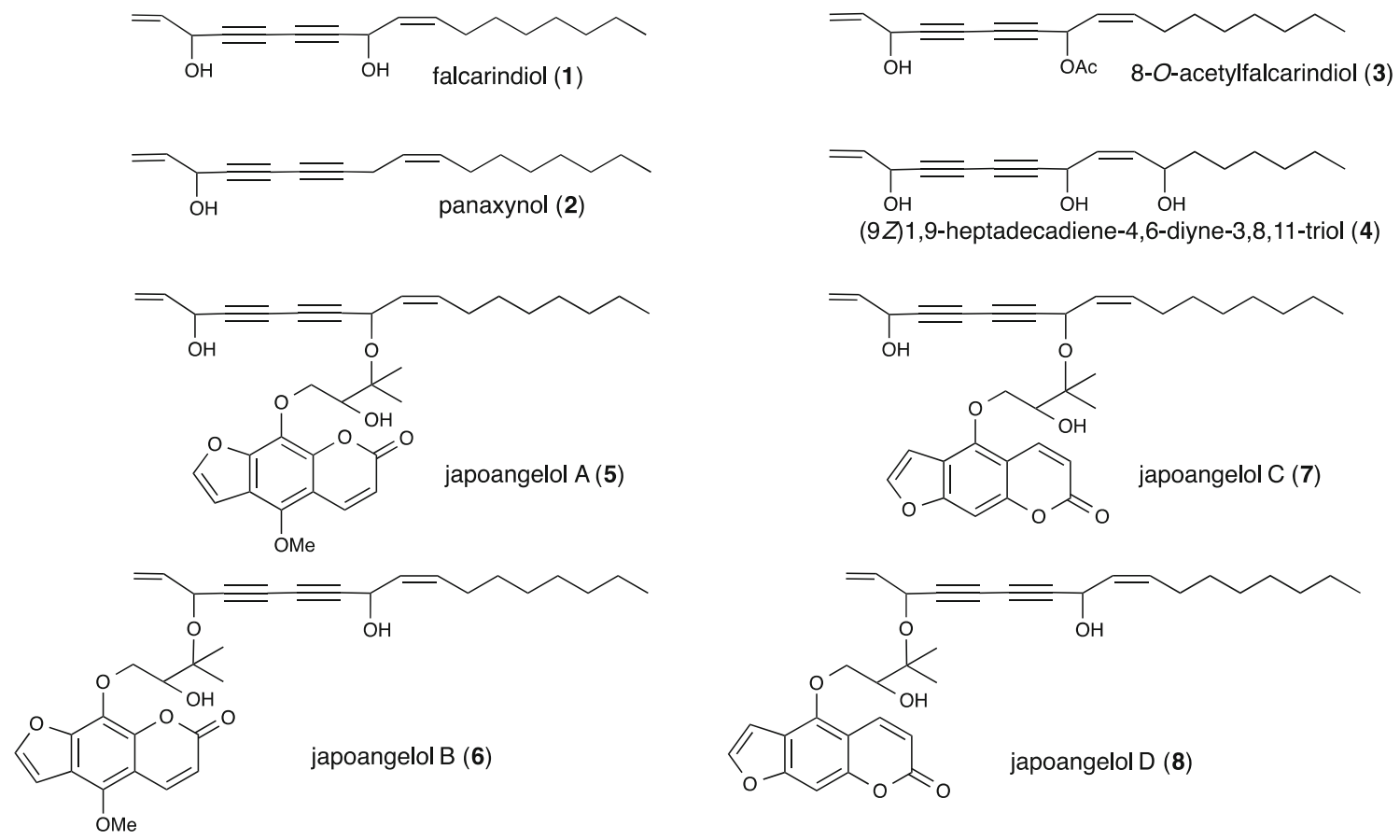

Fig. 1 Structures of polyacetylenes identified from a screen of plant extracts

from the leaves of Lantana montevidensis (Verbenaceae) [22]. Concurrently, several related flavones $(\mathbf{5 0}-\mathbf{5 7})$ isolated from other plant materials and two synthetic ones $(\mathbf{5 8}$, 59) were also evaluated. 5,7-Dihydroxy flavones $(\mathbf{3 9}, \mathbf{5 0}$, 51), 5,7-dihydroxy-6-methoxy flavones $(\mathbf{4 0}, \mathbf{4 1}, \mathbf{5 4}, \mathbf{5 5})$, and 6-methoxy flavone (59) were much stronger inhibitors of HeLa cell growth than B16F10 and MK-1 cell growth. In particular, compound $\mathbf{5 9}$ was a potent inhibitor of $\mathrm{HeLa}$ cell growth. Therefore, the 6-methoxy group is likely important for enhancing the anti-proliferative activity of flavones against HeLa cells. A synthetic flavone derivative, flavopiridol (Alvocidib), is being evaluated in clinical trials of ovarian and primary peritoneal cancers [23].

\section{Sesquiterpenes (Fig. 6)}

After activity-guided fractionation against MK-1, HeLa, and B16F10 cells, five active sesquiterpenes $(\mathbf{6 0 - 6 4})$ were isolated from the roots of Inula helenium (Asteraceae) together with an inactive sesquiterpene (65) and a weak one (66) [24]. A structure-activity study suggested that the presence of an $\alpha$-methylene- $\gamma$-lactone group is a key component required for the anti-proliferative activity. The thiol reactivity of the $\alpha$-methylene- $\gamma$-lactone group may be responsible for the observed anti-proliferative activity [25]. Two norsesquiterpene glycosides from the roots of Phyllanthus emblica (Phyllanthaceae) exhibited potent activity (data not shown) although their aglycone and monoglucoside showed no inhibitory activity [20].

\section{Triterpene glycosides (Fig. 7) and triterpenes}

\section{(Fig. 8)}

From the bioactive fraction of the fruits of Bupleurum rotundifolium (Apiaceae), ten ursane-type triterpene glycosides were isolated and their anti-proliferative activities against MK-1, HeLa, and B16F10 cells were estimated [26]. All active glycosides (67-71) have a 13ß, 28-epoxy 
Table 3 Anti-proliferative activities of compounds 1-118 $\left(\mathrm{EC}_{50}, \mu \mathrm{M}\right)$ against MK-1, HeLa, B16F10, MT-1, MT-2, and control PBMNC cells

\begin{tabular}{|c|c|c|c|c|c|c|}
\hline Compound name & MK-1 & HeLa & $\mathrm{B} 16 \mathrm{~F} 10$ & MT-1 & MT-2 & Normal \\
\hline Falcarindiol (1) & 15 & 149 & 89 & NT & NT & NT \\
\hline Panaxynol (2) & 1.2 & 224 & 80 & NT & NT & NT \\
\hline 8-O-Acetylfalcarindiol (3) & 274 & 175 & 203 & NT & NT & NT \\
\hline (9Z)1,9-Heptadecadiene-4,6-diyne-3,8,1 1-triol (4) & 8 & 106 & 62 & NT & NT & NT \\
\hline Japoangelol A (5) & 15 & 24 & 32 & NT & NT & NT \\
\hline Japoangelol B (6) & 8.7 & 26 & 42 & NT & NT & NT \\
\hline Japoangelol C (7) & 20 & 32 & 28 & NT & NT & NT \\
\hline Japoangelol D (8) & 30 & 41 & 53 & NT & NT & NT \\
\hline Deoxypodophyllotoxin (9) & 0.055 & 0.082 & 0.21 & 0.006 & 0.003 & NT \\
\hline (-)-Deoxypodorhizone (10) & 1.85 & 3.2 & 4 & NT & NT & NT \\
\hline Nemerosin (11) & 1.8 & 1 & 1.8 & NT & NT & NT \\
\hline Anthriscinol methyl ether (12) & 13 & 11 & 11 & NT & NT & NT \\
\hline Elemicin (13) & 22 & 9.6 & 13 & NT & NT & NT \\
\hline Anthriscusin (14) & 6.2 & 5.2 & 7.5 & NT & NT & NT \\
\hline Morelensin (15) & 0.24 & 0.14 & 0.23 & NT & NT & NT \\
\hline (-)-Hinokinin (16) & 4.8 & 7.3 & 7.6 & NT & NT & NT \\
\hline Acteoside (17) & 35 & 50 & 11 & NT & NT & NT \\
\hline Isoacteoside (18) & 40 & 32 & 10 & NT & NT & NT \\
\hline Arenarioside (19) & 34 & 34 & 16 & NT & NT & NT \\
\hline Leucosceptoside A (20) & 42 & 33 & 28 & NT & NT & NT \\
\hline Ligupurpuroside A (21) & 26 & 69 & 6.5 & NT & NT & NT \\
\hline Ligupurpuroside C (22) & 49 & 49 & 11 & NT & NT & NT \\
\hline Ligupurpuroside B (23) & $>135$ & $>135$ & 120 & NT & NT & NT \\
\hline Epicatechin (24) & 45 & NT & NT & NT & NT & NT \\
\hline Epigallocatechin (25) & 14 & NT & NT & NT & NT & NT \\
\hline Epigallocatechin gallate (26) & 9 & NT & NT & NT & NT & NT \\
\hline Gallocatechin (27) & 14 & NT & NT & NT & NT & NT \\
\hline Epicatechin gallate (28) & 14 & NT & NT & NT & NT & NT \\
\hline Gallocatechin gallate (29) & 10 & NT & NT & NT & NT & NT \\
\hline 7-O-Galloylcatechin $(\mathbf{3 0})$ & 41 & 38 & 9 & NT & NT & NT \\
\hline Gallic acid (31) & 19 & 22 & 7.1 & NT & NT & NT \\
\hline Gallic acid methylester (32) & 65 & 43 & 18 & NT & NT & NT \\
\hline Trigalloylgallic acid (33) & 10 & 9.3 & 2.9 & NT & NT & NT \\
\hline 1-O-Galloylglucose (34) & 60 & 45 & 15 & NT & NT & NT \\
\hline 1,6-Di- $O$-galloylglucose $(\mathbf{3 5})$ & 39 & 29 & 8.1 & NT & NT & NT \\
\hline Corilagin (36) & 13 & 30 & 4.7 & NT & NT & NT \\
\hline Prodelphidin B1 (37) & 13 & 15 & 3.3 & NT & NT & NT \\
\hline Prodelphidin B2 (38) & 15 & 15 & 3.3 & NT & NT & NT \\
\hline Apigenin (39) & 22 & 15 & 26 & NT & NT & NT \\
\hline Hispidulin (40) & 83 & 17 & 67 & NT & NT & NT \\
\hline Eupafolin (41) & 29 & 6 & 16 & NT & NT & NT \\
\hline Compound $\mathbf{4 2}$ & 55 & 55 & 18 & NT & NT & NT \\
\hline Compound $\mathbf{4 3}$ & 73 & 73 & 29 & NT & NT & NT \\
\hline Compound 44 & 33 & 44 & 39 & NT & NT & NT \\
\hline Cirsiliol (45) & 18 & 21 & 9 & NT & NT & NT \\
\hline Eupatorin (46) & 58 & 15 & 44 & NT & NT & NT \\
\hline Cirsilineol (47) & 17 & 203 & 73 & NT & NT & NT \\
\hline Compound 48 & 22 & 14 & 14 & NT & NT & NT \\
\hline
\end{tabular}


Table 3 continued

\begin{tabular}{|c|c|c|c|c|c|c|}
\hline Compound name & MK-1 & HeLa & B16F10 & MT-1 & MT-2 & Normal \\
\hline Compound 49 & $>267$ & $>267$ & 241 & NT & NT & NT \\
\hline Chrysin (50) & 63 & 8 & 51 & NT & NT & NT \\
\hline Luteolin(51) & 31 & 10 & 21 & NT & NT & NT \\
\hline Baicalein (52) & 26 & 30 & 11 & NT & NT & NT \\
\hline 6-Hydroxyluteolin (53) & 26 & 30 & 13 & NT & NT & NT \\
\hline Pectolinarigenin (54) & 115 & 10 & 64 & NT & NT & NT \\
\hline Desmethoxylcentaureidin (55) & 24 & 9 & 64 & NT & NT & NT \\
\hline Jaceosidin $(\mathbf{5 6})$ & 27 & 33 & 27 & NT & NT & NT \\
\hline Eupatilin (57) & 55 & 35 & 58 & NT & NT & NT \\
\hline 7-Methoxyflavone (58) & 119 & 87 & 119 & NT & NT & NT \\
\hline 6-Methoxyflavone (59) & 398 & 8 & 398 & NT & NT & NT \\
\hline 1,3,11(13)-Elematrien- $8 \beta, 12$-olide $(\mathbf{6 0})$ & 6.9 & 13 & 4.3 & NT & NT & NT \\
\hline $5 \alpha$-Epoxyalantolactone $(\mathbf{6 1})$ & 6.9 & 6.5 & 3.6 & NT & NT & NT \\
\hline $4 \beta, 5 \alpha$-epoxy-1(10),11(13)-germacradiene-8,12-olide (62) & 12 & 33 & 14 & NT & NT & NT \\
\hline Alantolactone (63) & 6.9 & 6.9 & 4.7 & NT & NT & NT \\
\hline Isoalantolactone (64) & 44 & 41 & 29 & NT & NT & NT \\
\hline $11 \alpha, 13$-Dihydroalantolactone $(\mathbf{6 5})$ & $>427$ & $>427$ & $>427$ & NT & NT & NT \\
\hline $11 \alpha, 13$-Dihydroisoalantolactone (66) & $>427$ & $>427$ & 44 & NT & NT & NT \\
\hline Rotundifolioside I (67) & 20 & 37 & 18 & NT & NT & NT \\
\hline Rotundifolioside J (68) & 16 & 21 & 11 & NT & NT & NT \\
\hline Rotundifolioside A (69) & 48 & 71 & 31 & NT & NT & NT \\
\hline Rotundifolioside H (70) & 18 & 31 & 18 & NT & NT & NT \\
\hline Rotundifolioside G (71) & 84 & $>108$ & 46 & NT & NT & NT \\
\hline Rotundifolioside E (72) & $>110$ & $>110$ & $>110$ & NT & NT & NT \\
\hline Rotundifolioside F (73) & $>108$ & $>108$ & $>108$ & NT & NT & NT \\
\hline Rotundioside F (74) & 17 & 19 & 6.6 & NT & NT & NT \\
\hline Rotundioside G (75) & 7.8 & 15 & 17 & NT & NT & NT \\
\hline Rotundioside T (76) & 13 & 12 & 7.7 & NT & NT & NT \\
\hline Rotundioside Q (77) & 34 & 37 & 12 & NT & NT & NT \\
\hline Rotundioside S (78) & 19 & 34 & 8.9 & NT & NT & NT \\
\hline Ursolic acid lactone (79) & 90 & 88 & 194 & NT & NT & NT \\
\hline Ursolic acid $(\mathbf{8 0})$ & 19 & 65 & 14 & NT & NT & NT \\
\hline Pomolic acid (81) & 55 & 59 & 29 & NT & NT & NT \\
\hline Corosolic acid (82) & 59 & 69 & 44 & NT & NT & NT \\
\hline $2 \alpha, 3 \alpha$-Dihydoxy-urs-12-en-28-oic acid (83) & 55 & 38 & 36 & NT & NT & NT \\
\hline 3-Epimaslinic acid (84) & 21 & 21 & 19 & NT & NT & NT \\
\hline Psoralen $(\mathbf{8 5})$ & 403 & 40 & 376 & 345 & 177 & NT \\
\hline Bergapten (86) & 167 & 37 & 167 & 189 & 214 & NT \\
\hline Xanthotoxol (87) & $>431$ & 16 & 289 & NT & NT & NT \\
\hline 8-Hydroxybergapten $(\mathbf{8 8})$ & 139 & 8.9 & 104 & NT & NT & NT \\
\hline Xanthotoxin (89) & 139 & 74 & 181 & 73 & 48 & NT \\
\hline Isopimpinellin (90) & 151 & 53 & 159 & 85 & 231 & NT \\
\hline $\begin{array}{l}\text { 1,3-Dihydroxy-4-(2'-hydroxy-3'- } \\
\text { hydroxymethyl-3',4'-epoxybutyl)- } \\
N \text {-methylacridone (91) }\end{array}$ & 0.056 & 0.056 & 1.76 & NT & NT & NT \\
\hline $\begin{array}{l}\text { 1,3-Dihydroxy-4-[(Z)-3'-hydroxy-3'- } \\
\text { methylbuten-1'-yl]- } N \text {-methylacridone (92) }\end{array}$ & 308 & 68 & 13 & NT & NT & NT \\
\hline 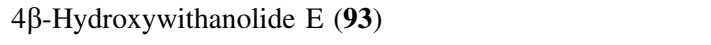 & NT & NT & NT & 0.2 & 0.2 & 1.6 \\
\hline withanolide S (94) & NT & NT & NT & 196 & 81 & NT \\
\hline
\end{tabular}


Table 3 continued

\begin{tabular}{|c|c|c|c|c|c|c|}
\hline Compound name & MK-1 & HeLa & B16F10 & MT-1 & MT-2 & Normal \\
\hline $5 \alpha$-O-Methylwithanolide S (95) & NT & NT & NT & 21 & 3.6 & NT \\
\hline $5 \alpha$-O-Butylwithanolide $\mathrm{S}(\mathbf{9 6})$ & NT & NT & NT & 2.4 & 0.8 & NT \\
\hline 2-Hydro-3 $\beta$-methoxy-4 $\beta$-hydroxywithanolide E (97) & NT & NT & NT & 1.9 & 1.7 & NT \\
\hline Sitoindoside IX (98) & NT & NT & NT & 0.83 & 6.1 & NT \\
\hline Withaferine A (99) & NT & NT & NT & 0.16 & 1.3 & NT \\
\hline 2,3-Dihydrowithaferine A (100) & NT & NT & NT & 0.022 & 0.51 & NT \\
\hline 24,25-Dihydrowithanolide D (101) & NT & NT & NT & 0.008 & 0.008 & 860 \\
\hline Physapruin A (102) & NT & NT & NT & 0.05 & 0.28 & NT \\
\hline Withanolide F (103) & NT & NT & NT & 1.4 & 1.6 & NT \\
\hline Nivaphysalin A (104) & NT & NT & NT & $>100$ & $>100$ & NT \\
\hline Nivaphysalin B (105) & NT & NT & NT & $>100$ & $>100$ & NT \\
\hline Nivaphysalin C (106) & NT & NT & NT & 59 & 58 & NT \\
\hline Liriodenine (107) & NT & NT & NT & 3.1 & 3.6 & NT \\
\hline Lysicamne (108) & NT & NT & NT & 32 & 16 & NT \\
\hline Lanuginosine (109) & NT & NT & NT & 1.3 & 4.5 & NT \\
\hline $14 \beta$-Hydroxytylophorine $N$-oxide (110) & NT & NT & NT & 0.07 & 0.027 & NT \\
\hline Tylophorinine $N$-oxide (111) & NT & NT & NT & 0.029 & 0.0048 & NT \\
\hline 3-Demethyl- $14 \alpha$-hydroxyisotylocrebrine $N$-oxide (112) & NT & NT & NT & 0.0083 & 0.0071 & 0.04 \\
\hline Tylophorine $N$-oxide (113) & NT & NT & NT & 1.6 & 1.5 & NT \\
\hline Isotylocrebrine $N$-oxide (114) & NT & NT & NT & 0.38 & 0.25 & NT \\
\hline 3-Demethyl-14 $\beta$-hydroxyisotylocrebrine (115) & NT & NT & NT & 0.0028 & 0.0026 & NT \\
\hline Tylophorine (116) & NT & NT & NT & 0.076 & 0.051 & NT \\
\hline Isotylocrebrine (117) & NT & NT & NT & 0.048 & 0.025 & NT \\
\hline 7-Demethyltylophorine (118) & NT & NT & NT & 0.019 & 0.029 & NT \\
\hline $5-\mathrm{FU}$ & 21 & 13 & 1.1 & NT & NT & NT \\
\hline DOX & NT & NT & NT & 0.015 & 0.013 & NT \\
\hline
\end{tabular}

Dox doxorubicin, 5-FU 5-fluorouracil (positive controls), NT not tested<smiles>[R]c1cc([C@H]2c3cc4c(cc3C[C@H]3COC(=O)[C@H]23)OCO4)cc(OC)c1OC</smiles>

deoxypodophyllotoxin (9) morelensin (15) $\mathrm{H}$<smiles>COc1cc(CC2C(=O)OCC2Cc2ccc3c(c2)OCO3)cc(OC)c1OC</smiles>

(-)-deoxypodorhizone (10) $\mathrm{OMe}$<smiles>COC/C=C/c1cc(OC)c2c(c1)OCO2</smiles>

anthriscinol methyl ether (12)<smiles>C=CCc1cc(OC)c(OC)c(OC)c1</smiles>

elemicin (13)<smiles>O=C1OC[C@H](Cc2ccc3c(c2)OCO3)[C@H]1Cc1ccc2c(c1)OCO2</smiles>

(-)-hinokinin (16)<smiles>COc1cc(/C=C2/C(=O)OC[C@H]2Cc2ccc3c(c2)OCO3)cc(OC)c1OC</smiles>

nemerosin (11)<smiles>CC=C(COC(=O)C/C(C)=C\C)C(=O)OC/C=C/c1cc(OC)c2c(c1)OCO2</smiles>

Fig. 2 Structures of lignans identified from a screen of plant extracts 
Fig. 3 Structures of phenylethanoides identified from a screen of plant extracts

\section{acteoside (17) \\ ligupurpuroside $A(\mathbf{2 1})$}

ligupurpuroside C (22)

ligupurpuroside B (23)

isoacteoside (18)

arenarioside

(19)

leucosceptoside $A(\mathbf{2 0})$

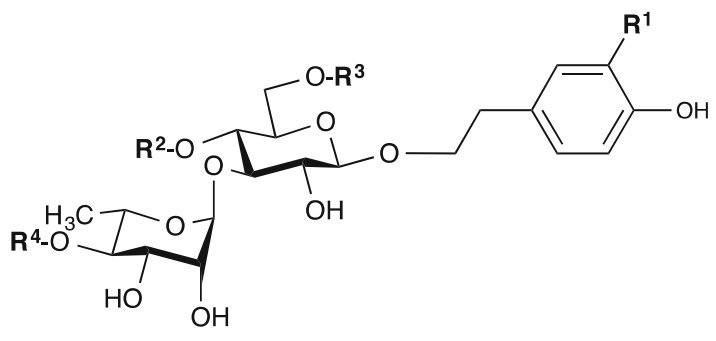

(n)

ring system in the molecule except for $\mathbf{7 2}$ and $\mathbf{7 3}$, which have a $21 \beta$-hydroxy group. The glycosides of the other aglycones are almost inactive. Among the active glycosides, 69 and 71, which have a glucosyl group directly linked to the aglycone instead of a fucosyl group, were less potent. It is possible that the fucosyl group plays some role in the anti-proliferative activity. From the same fraction, 19 oleanane-type triterpene glycosides were also isolated and their anti-proliferative activities were evaluated [27]. Similar to the ursane-type triterpene glycosides, all active glycosides (74-78) have a 13 $\beta, 28$-epoxy ring system in the molecule. In contrast to the ursane-type triterpene glycosides, compounds $\mathbf{7 7}$ and $\mathbf{7 8}$, which have a $21 \alpha$-hydroxy group, had potent anti-proliferative activities. The configuration at the C-21 hydroxy group might influence the antiproliferative activity.

After activity-guided fractionation against MK-1, HeLa and $\mathrm{B} 16 \mathrm{~F} 10$ cells, ten triterpenes were isolated from the aerial parts of Centella asiatica (Apiaceae) [28]. Some (7984) of these triterpenes showed potent anti-proliferative activities. Similar to the results of the polyphenols, ursolic acid $(\mathbf{8 0})$ with a free carboxyl group showed higher activity than its lactone (79). Ursolic acid (80) was previously reported to induce apoptoic cell death [29].

\section{Coumarins and acridone alkaloids (Fig. 9)}

After activity-guided fractionation against MK-1, HeLa, and B16F10 cells, 16 compounds were isolated from the aerial parts and roots of Boenninghausenia japonica $(\mathrm{Ru}-$ taceae) [30]. Among them, an acridone alkaloid (91) showed very strong anti-proliferative activity against these three cell lines. The $\mathrm{EC}_{50}$ value of $\mathbf{9 1}$ was in the nanomolar range except for against B16F10 cells. Therefore, a $3^{\prime}, 4^{\prime}$ epoxy group might be important for enhancing the antiproliferative activity of acridone alkaloids. Furthermore, some furanocoumarins (85-90) showed potent anti-proliferative activities against HeLa cells. The furanocoumarins $(\mathbf{8 7}, \mathbf{8 8})$ having an 8-hydroxy group showed more potent activity than those without the substituent $(\mathbf{8 5}, \mathbf{8 6})$ and those with an 8-methoxy group $(\mathbf{8 9}, \mathbf{9 0})$ against HeLa cells. Therefore, an 8-hydroxy group may be important for enhancing the anti-proliferative activity of these compounds against HeLa cells. Some furanocoumarins $(\mathbf{8 5}, \mathbf{8 6}$, 
Fig. 4 Structures of polyphenols identified from a screen of plant extracts<smiles>[R1]O[C@@H]1Cc2c(O)cc(O)cc2O[C@@H]1c1cc([R1])c(O)c(O)c1</smiles>

epicatechin (24) epigallocatechin (25) epigallocatechin gallate (26) epicatechin gallate (28)<smiles>[R][C@H]1Cc2c(O)cc(O)c([C@@H]3c4c(O)cc(O)cc4O[C@H](c4cc(O)c(O)c(O)c4)[C@H]3O)c2O[C@H]1c1cc(O)c(O)c(O)c1</smiles><smiles>[R2]Oc1cc(O)c2c(c1)O[C@H](c1cc([R3])c(O)c(O)c1)[C@@H](O)C2</smiles>

gallocatechin (27) 7-O-galloylcatechin (30) $\mathrm{G}$ H
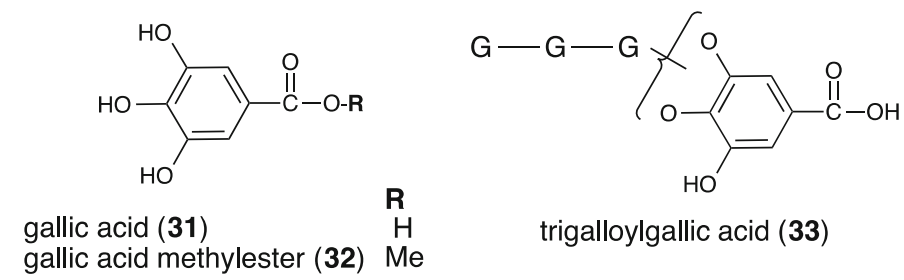

trigalloylgallic acid (33)

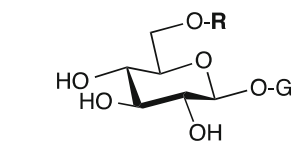

1-O-galloylglucose (34) 1,6-di- $O$-galloylglucose (35) G

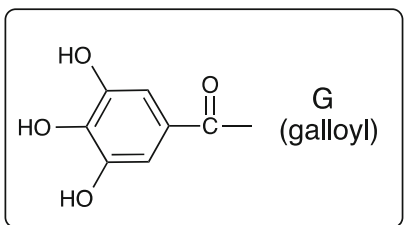

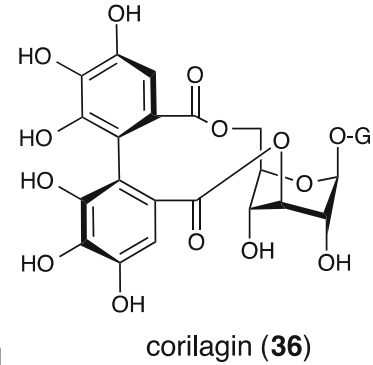

corilagin (36)
89, 90) also showed moderate anti-proliferative activity against MT-1 and MT-2 cells (Table 3). A recent review reports that natural and synthetic coumarins have anticancer activity toward various cell lines [31].

\section{Withanolides (Fig. 10)}

After activity-guided fractionation against MT-1 and MT-2 cells, five active withanolides (93-97) were isolated from the aerial parts of Physalis pruinosa (Solanaceae) [32]. Structure-activity relationships suggested that the presence of a $5 \beta, 6 \beta$ epoxy group in the B-ring and a $4 \beta$-hydroxy group in the A-ring were important for the observed activities. The aliphatic ether side chain at C-5 also seems to increase the activity because as the side chain is lengthened, the activity increases. Because the $\mathrm{EC}_{50}$ value for $4 \beta$-hydroxywithanolide $\mathrm{E}$ (93) was in the nanomolar range against both MT-1 and MT-2 cells, 31 other withanolides were also evaluated [33]. Except for compound 98, none of the glycosides showed any activity against the ATL cell lines. Because compound $\mathbf{9 8}$ has a $5 \beta, 6 \beta$-epoxy group as well as a $4 \beta$-hydroxy group, we predicted it might show potent activity. However, the activity of the corresponding deglycosylated compound (99) was approximately four times greater than that of its glucoside (98). These results indicated that the presence of a sugar moiety should reduce the anti-proliferative effects. The importance of the $5 \beta, 6 \beta$-epoxy group, and $4 \beta$-hydroxy group was further supported by the analysis of compound $\mathbf{1 0 0}$ because it showed the second strongest anti-proliferative activity. The activities of 99, having a double bond between C-2 and $\mathrm{C}-3$, were weaker than those for $\mathbf{1 0 0}$, suggesting the double bond between $\mathrm{C}-2$ and $\mathrm{C}-3$ might reduce the activity. Because compound $\mathbf{1 0 2}$ showed greater activity when compared with compound $\mathbf{1 0 3}$, the importance of a $4 \beta$ hydroxy group was further supported. Compared with the compounds having the $5 \beta, 6 \beta$-epoxy group, the activities of the compounds (104-106) having other types of epoxy groups $(6 \alpha, 7 \alpha$-epoxy group in the B-ring and/or $24 \alpha, 25 \alpha$ epoxy group in the E-ring) were significantly lower. The position of the epoxy group and/or the configuration of the epoxy group seem to be important for the activity. Compound 106 containing a $15 \beta$-hydroxy group showed moderate activity while compound $\mathbf{1 0 5}$ containing a $15 \alpha$ - 


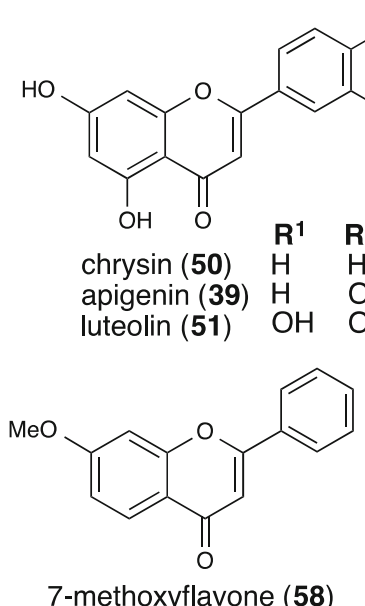<smiles>COc1ccc2oc(-c3ccccc3)cc(=O)c2c1</smiles>

6-methoxyflavone (59)<smiles>[R1][R1]#[R]</smiles>

$\begin{array}{lll}\text { baicalein (52) } & \stackrel{H}{\mathbf{H}^{1}} & \stackrel{\mathrm{H}}{\mathbf{2}^{2}} \\ \text { 6-hydroxyluteolin (53) } & \mathrm{OH} & \mathrm{OH}\end{array}$<smiles>[R1]c1cc(-c2cc(=O)c3c(O)c(O)c(OC)cc3o2)cc(OCC)c1OCC</smiles>

$\begin{array}{llll} & \mathbf{R}^{\mathbf{1}} & \mathbf{R}^{\mathbf{2}} & \mathbf{R}^{\mathbf{3}} \\ \mathbf{4 2} & \mathrm{H} & \mathrm{Me} & \mathrm{H} \\ \mathbf{4 3} & \mathrm{Me} & \mathrm{Me} & \mathrm{H} \\ \mathbf{4 4} & \mathrm{Me} & \mathrm{H} & \mathrm{OMe}\end{array}$<smiles>[R1]C=[R1]</smiles>

hispidulin (40) pectolinarigenin (54) eupafolin (41) desmethoxylcentaureidin (55) OH $\mathrm{H}$ jaceosidin (56) OMe $\mathrm{H}$ eupatilin (57) $\quad \mathrm{OMe} \mathrm{Me}$<smiles>[R]c1cc(-c2cc(=O)c3c(O)c(OC)c(OC)cc3o2)cc([R])c1[R]</smiles>

$\begin{array}{lll}\mathbf{R}^{1} & \mathbf{R}^{2} & \mathbf{R}^{3}\end{array}$

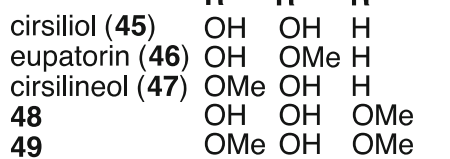

Fig. 5 Structures of flavones identified from a screen of plant extracts as well as those derived synthetically

Fig. 6 Structures of sesquiterpenes identified from a screen of plant extracts

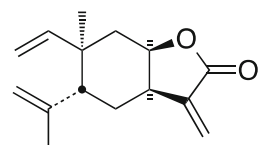

1,3,11(13)-elematrien-8 $\beta, 12$-olide (60)<smiles>C=C1C(=O)O[C@H]2C[C@@]3(C)CCCC(C)C3=C[C@H]12</smiles>
alantolactone $(63)$<smiles>C=C1CCC[C@]2(C)C[C@H]3OC(=O)[C@H](C)[C@H]3CC12</smiles>

$11 \alpha, 13$-dihydroisoalantolactone<smiles>C=C1C(=O)O[C@H]2C[C@@]3(C)CCC[C@H](C)[C@@]34O[C@H]4[C@H]12</smiles>

$5 \alpha$-epoxyalantolactone (61)<smiles>C=C1C(=O)O[C@H]2C[C@@]3(C)CCCC(=C)C3CC12</smiles>

isoalantolactone $(64)$<smiles>C=C1C(=O)O[C@@H]2C/C3=C/CC[C@]4(C)O[C@]4(CC3)C12</smiles>

$4 \beta, 5 \alpha$-epoxy-1(10),11(13)germacradiene-8,12-olide (62)<smiles>CC1CCC[C@]2(C)C[C@H]3OC(=O)[C@H](C)[C@H]3C=C12</smiles>

$11 \alpha, 13$-dihydroalantolactone (65) hydroxy group did not show any activity. This indicated that the configuration of the hydroxy group at C-15 may influence a compound's activity. Finally, the $\mathrm{EC}_{50}$ value of 24, 25-dihydrowithanolide D (101), the most potent withanolide-type inhibitor, was $8 \mathrm{nM}$ against both cells. In contrast, the cytotoxic effect $(860 \mathrm{nM})$ of $\mathbf{1 0 1}$ toward normal cells was observed at a concentration about 100 times higher than those observed for the ATL cell lines. Furthermore, compound $\mathbf{1 0 1}$ was confirmed to induce dose-dependent apoptosis against MT-1, MT-2, and fresh
ATL cells [33]. Therefore, 24, 25-dihydrowithanolide D (101) may be a promising chemotherapeutic candidate.

Recently inhibition of the growth of human lung cancer cells through DNA damage, apoptosis and G2/M arrest by $4 \beta$-hydroxywithanolide $\mathrm{E}$ (93) have been reported [34]. Further, induction of apoptosis in leukemia cells by targeting the activation of a neutral sphingomyelinase-ceramide cascade mediated by synergistic activation of c-Jun N-terminal kinase and p38 mitogen-activated protein kinase by withanolide $\mathrm{D}$ have been also reported [35]. 
Fig. 7 Structures of triterpene glycosides identified from a screen of plant extracts

Fig. 8 Structures of triterpenes identified from a screen of plant extracts

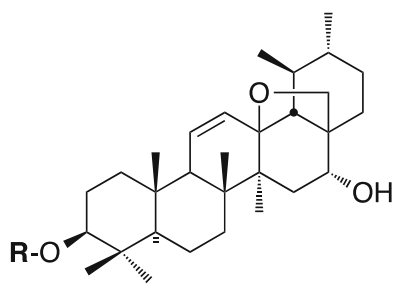

rotundifolioside I (67) : R = Fuc ${ }^{2}-^{1} \mathrm{Glc}^{2}{ }^{1}{ }^{1} \mathrm{Xyl}$

rotundifolioside $\mathrm{J}(68): \mathrm{R}=\mathrm{Fuc}^{2}{ }^{1} \mathrm{Glc}^{2}{ }^{-1} \mathrm{Rha}$ rotundifolioside $A(69): R=G^{2} c^{2}{ }^{1} G c^{2}{ }^{1} X y l$

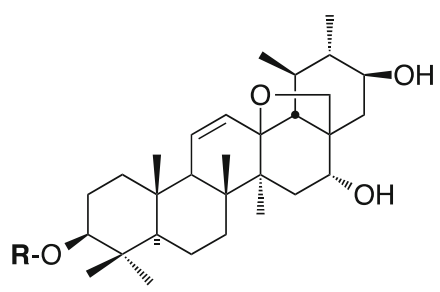

rotundifolioside $E(72): R=F^{2}{ }^{2}{ }^{1} G l^{2}{ }^{1} X y l$ rotundifolioside $F(73): R=$ Fuc $^{2}{ }^{1}{ }^{G} \mathrm{Gl}^{2}{ }^{1} \mathrm{Rha}$

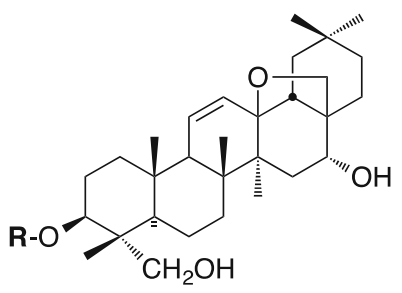

rotundioside $\mathrm{T}(\mathbf{7 6}): \mathrm{R}=\mathrm{Fuc}^{2} \mathrm{-}^{-1} \mathrm{G} \mid \mathrm{c}^{2}{ }^{-1} \mathrm{Xyl}$

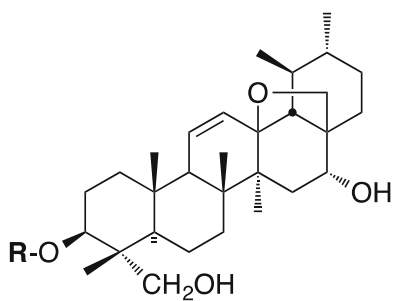

rotundifolioside $\mathrm{H}(\mathbf{7 0}): \mathrm{R}=\mathrm{Fuc}^{2}{ }^{1} \mathrm{Glc}^{2}{ }^{1} \mathrm{Xyl}$

rotundifolioside $\mathrm{G}(\mathbf{7 1})$ : $\mathrm{R}=\mathrm{Glc}^{2} \_^{1} \mathrm{G} \mid \mathrm{c}^{2}{ }^{1} \mathrm{Xyl}$

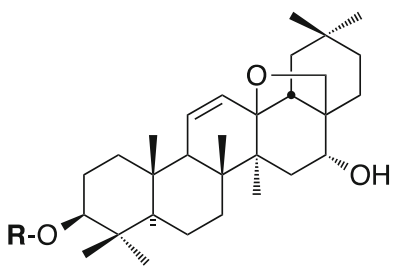

rotundioside $F(74): R=F_{c}^{2}{ }^{1} G_{l}{ }^{2}{ }^{1} R$ ha rotundioside $\mathrm{G}(\mathbf{7 5})$ : $\mathrm{R}=\mathrm{Fuc}^{2}{ }^{-1} \mathrm{Glc}^{2}{ }^{-1} \mathrm{Xyl}$

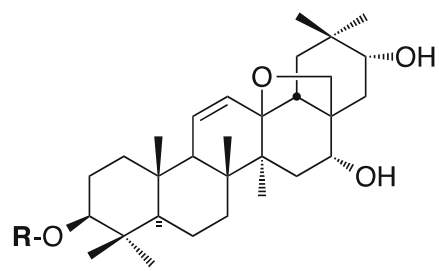

rotundioside $Q(77): R=$ Fuc $^{2}{ }^{1}{ }^{1} \mathrm{Glc}^{2}{ }^{2}{ }^{1} \mathrm{Xyl}$ rotundioside $S(78): R=$ Fuc $^{2}{ }^{1} \mathrm{Glc}^{2}{ }^{1}$ Rha

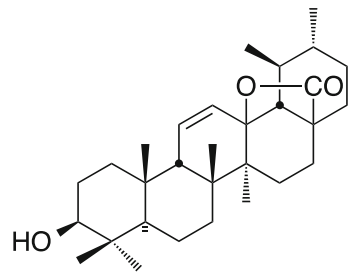

ursolic acid lactone (79)<smiles>C[C@H]1CC[C@]2(C(=O)O)CC[C@]3(C)C(=CC[C@@H]4[C@@]5(C)CC[C@H](O)C(C)(C)[C@@H]5CC[C@]43C)C2[C@@H]1C</smiles>

ursolic acid (80)<smiles>C[C@H]1CC[C@]2(C(=O)O)CC[C@]3(C)C(=CC[C@@H]4[C@@]5(C)CC[C@H](O)C(C)(C)[C@@H]5CC[C@]43C)[C@@H]2[C@@]1(C)O</smiles>

pomolic acid (81)

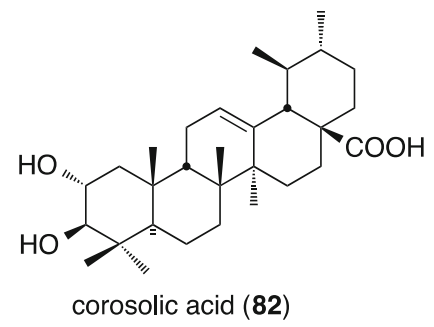

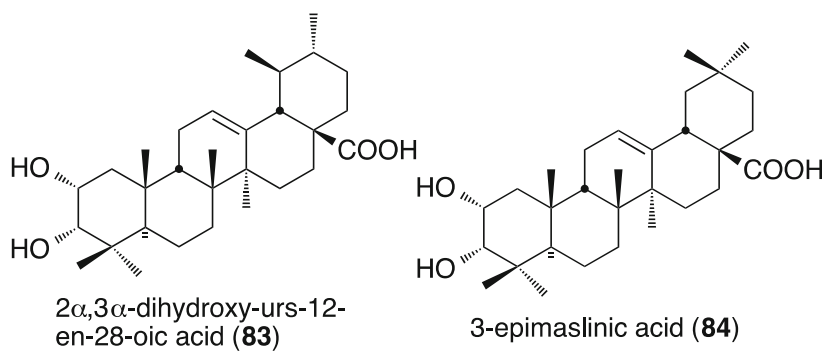


Fig. 9 Structures of coumarins and acridone alkaloids identified from a screen of plant extracts

Fig. 10 Structures of withanolides identified from a screen of plant extracts<smiles>O=c1ccc2cc3ccoc3cc2o1</smiles><smiles>COc1c2ccoc2cc2oc(=O)ccc12</smiles>

psoralen (85)<smiles>O=c1ccc2cc3ccoc3c(O)c2o1</smiles>
xanthotoxol (87)<smiles>COc1c2occc2cc2ccc(=O)oc12</smiles>
xanthotoxin (89)

$$
\text { bergapten (86) }
$$<smiles>COc1c2ccoc2c(O)c2oc(=O)ccc12</smiles>

8-hydroxybergapten (88)<smiles>COc1c2ccoc2c(OC)c2oc(=O)ccc12</smiles>
isopimpinellin (90)<smiles>Cn1c2ccccc2c(=O)c2c(O)cc(O)c(CC(O)C3(CO)CO3)c21</smiles>

1,3-dihydroxy-4-(2'-hydroxy-3'-hydroxymethyl3',4'-epoxybutyl)- $N$-methylacridone (91)<smiles>Cn1c2ccccc2c(=O)c2c(O)cc(O)c(/C=C\C(C)(C)O)c21</smiles>

1,3-dihydroxy-4-[(Z)-3'-hydroxy-3'-methylbuten1 '-yl]- $N$-methylacridone (92)

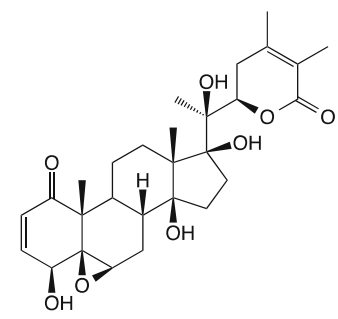

43-hydroxywithanolide E (93)

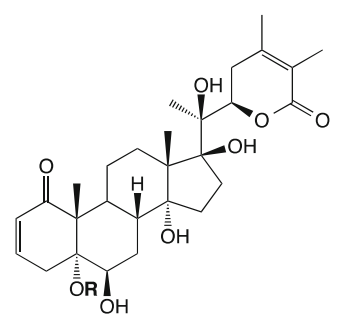

withanolide $\mathrm{S}$ (94) : $\mathbf{R}=\mathrm{H}$ $5 \alpha$-O-methylwithanolide $\mathrm{S}(95): \mathbf{R}=\mathrm{CH}_{3}$ $5 \alpha$-O-butylwithanolide $S(96): \mathbf{R}=n$-Butyl

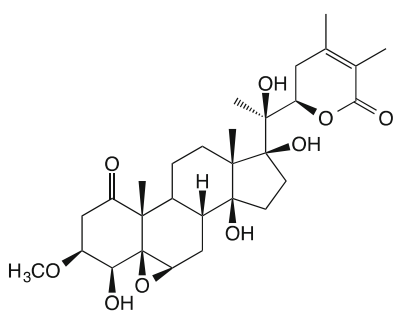

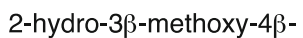
hydroxywithanolide $E$ (97)

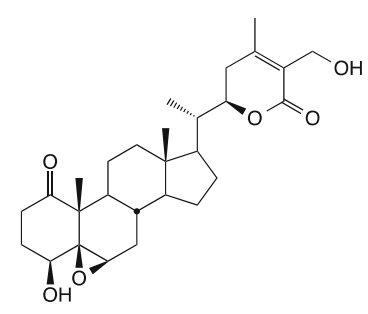

2,3-dihydrowithaferine $A(\mathbf{1 0 0})$

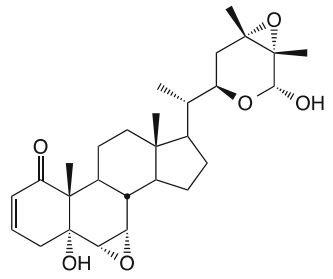

nivaphysalin $A$ (104)

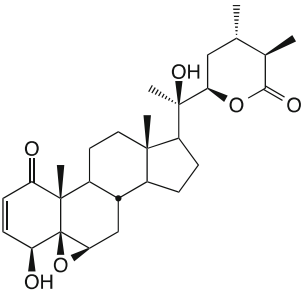

24,25-dihydrowithanolide D (101) withaferine $A(99): R=H$

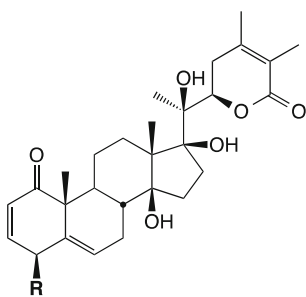

physapruin $\mathrm{A}(\mathbf{1 0 2}): \mathbf{R}=\mathrm{OH}$ withanolide $\mathrm{F}(\mathbf{1 0 3}): \mathbf{R}=\mathrm{H}$

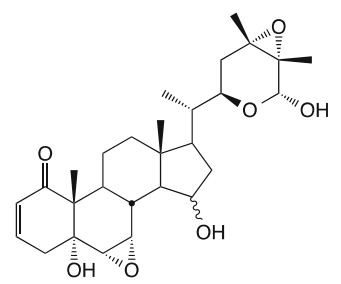

nivaphysalin $B(105): 15 \alpha$ nivaphysalin C (106) : $15 \beta$ 
Fig. 11 Structures of aporphine and phenanthroindolizidine alkaloids identified from a screen of plant extracts<smiles>O=C1c2ccccc2-c2c3c(cc4ccnc1c24)COC3</smiles>

Liriodenine (107)<smiles>COc1cc2ccnc3c2c(c1OC)-c1ccccc1C3=O</smiles>

Lysicamine (108)<smiles>COc1ccc2c(c1)C(=O)C1=NC=Cc3cc4c(c1c3-2)COC4</smiles>

Lanuginosine (109)<smiles></smiles>

$14 \beta$-hydroxytylophorine $N$-oxide (110)<smiles>COc1cc2c3c(c4cc(OC)c(OC)cc4c2cc1OC)C1CCC[N+]([O-])(C3)C1</smiles>

tylophorine $N$-oxide (113)<smiles>COc1cc2c3c(c4cc(OC)c(OC)cc4c2cc1OC)CN1CCCC1C3</smiles>

tylophorine (116)<smiles>COc1ccc2c3c(c4cc(OC)c(OC)cc4c2c1)C[N@@+]1([O-])CCC[C@H]1[C@H]3O</smiles>

tylophorinine $N$-oxide (111)<smiles>COc1cc2c3c(c4ccc(OC)c(OC)c4c2cc1OC)C[C@@]1(O)CCC[C@H]1C3</smiles>

isotylocrebrine $N$-oxide (114)<smiles>COc1cc2c3c(c4ccc(OC)c(OC)c4c2cc1OC)CC1CCCN1C3</smiles>

isotylocrebrine (117)<smiles>COc1cc2c3c(c4ccc(O)c(OC)c4c2cc1OC)[C@@H](O)[C@H]1CCCN1O3</smiles>

3-demethyl-14 $\alpha$ hydroxyisotylocrebrine $N$-oxide (112)<smiles>COc1cc2c3c(c4ccc(O)c(OC)c4c2cc1OC)[C@@H](O)C1CCCN1C3</smiles>

3-demethyl-14 $\beta$ hydroxyisotylocrebrine (115)<smiles></smiles>

7-demethyltylophorine (118)

Wang et al. suggested that Hsp90 inhibition by the withanolides is correlated with their ability to induce cancer cell death [36].

\section{Aporphine and phenanthroindolizidine alkaloids}

(Fig. 11)

After activity-guided fractionation against MT-1 and MT-2 cells, three active aporphine alkaloids (107-109)

were isolated from the leaves of Annona reticulata and A. squamosa (Annonaceae) [37]. Liriodenine (107) showed accumulation of Sub-G1 stage cells in the MT-1 and MT-2 cell population, suggesting induction of apoptosis. A structure-activity relationship analysis suggested that the presence of a 1, 2-methylenedioxy group seemed to enhance activity. A similar conclusion on the structure-activity relationship was also obtained by Liu et al. [38]. 
Fig. 12 Differences in the specific selectivity of selected compounds against various cancer cell lines
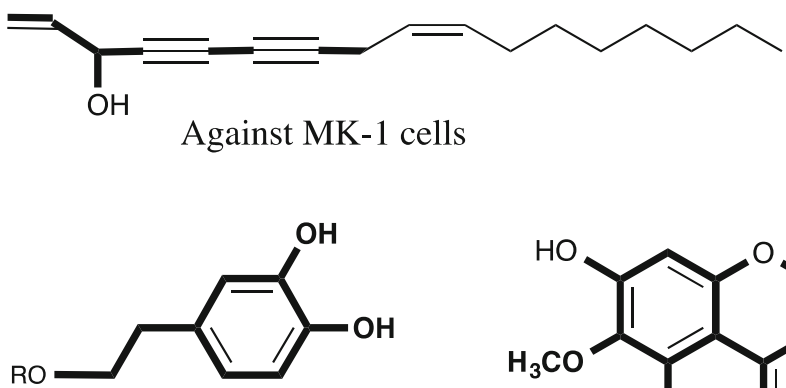<smiles>COc1c(O)cc2oc(-c3ccccc3)cc(=O)c2c1O</smiles>

Against B16F10 cells

Against HeLa cells
Six phenanthroindolizidine alkaloids (110-115) were isolated from the aerial parts of Tylophora tanakae (Asclepiadaceae) by activity-guided fractionation [39]. In addition to 110-115, three phenanthroindolizidine alkaloids (116-118) obtained from other plants were examined for their anti-proliferative activity against MT-1 and MT-2 cells. The $\mathrm{EC}_{50}$ values of all alkaloids except for compound 113 were in the low nanomolar range. The results suggested that the presence of a 2-methoxy functionality, the methyl group of a 7-methoxy functionality, and an $\mathrm{N}$-oxide moiety appear to reduce the potency of the anti-proliferative activity [39]. Phenanthroindolizidine alkaloids are cytotoxic to multidrug-resistant cells [40], inhibiting the enzyme dihydrofolate reductase [41]. The in vivo efficacy of a new phenanthroindolizidine alkaloid derivative (YPC10157) was recently evaluated [42].

\section{Conclusions}

Cytotoxicity against selected cancer cell lines was characterized and could be explained by identifying the active principles responsible for the observed effects. The polyacetylenes was more potent against MK-1 cells than against HeLa and $\mathrm{B} 16 \mathrm{~F} 10$ cells. The $\mathrm{EC}_{50}$ value of the most potent polyacetylene (2) against MK-1 cells was $1.2 \mu \mathrm{M}$ (Fig. 12). The compounds (17-22) having a 3, 4-dihydroxyphenethyl group also showed remarkable antiproliferative effects against B16F10 cells (Fig. 12). Interestingly, some 6-methoxyflavone derivatives $(\mathbf{4 0}, \mathbf{4 1}, \mathbf{5 4}$, $\mathbf{5 5}, \mathbf{5 9})$ and 8-hydroxy furanocoumarins $(\mathbf{8 7}, \mathbf{8 8})$ showed strong inhibition against HeLa cell growth (Fig. 12).

The compounds whose $\mathrm{EC}_{50}$ values were less than one nanomolar $(<1 \mathrm{nM})$ were not selective for specific cell types. This group included two lignans $(\mathbf{9}, \mathbf{1 5})$, one acridone alkaloid (91), six withanolides $(\mathbf{9 3}, \mathbf{9 8}-\mathbf{1 0 2})$, and eight phenanthroindolizidine alkaloids (110-112, 114118). Because the cytotoxic effect of 24,25 -dihydrowithanolide D (101) toward normal cells was observed at a concentration about 100 times higher than against the ATL cell lines, withanolide was concluded to be the most promising chemotherapeutic candidate from our experiments.

Acknowledgments We thank Drs. Tsuneatsu Nagao, Fumiko Abe, Kunihide Mihashi, Hisashi Matsunaga, Ryuji Ikeda, Takashi Tanaka, Tenji Konishi, Kenji Ishitsuka, Ryota Tsuchihashi, and Masafumi Okawa for supporting our work.

\section{References}

1. Lee KH (2010) Discovery and development of natural productderived chemotherapeutic agents based on a medicinal chemistry approach. J Nat Prod 73:500-516

2. Balunas MJ, Kinghorn AD (2005) Drug discovery for medicinal plants. Life Sci 78:431-441

3. Cragg GM, Newman DJ (2005) Plant as a source of anti-cancer agents. J Ethnopharmacol 100:72-79

4. Nagao T, Kinjo J, Okabe H (2004) Anti-proliferative phenolic constituents in plants and structure-activity relationships. FFI J 209:2-12

5. Gandhi V, Plunkett W, Cortes JE (2014) Omacetaxine: a protein translation inhibitor for treatment of chronic myelogenous leukemia. Clin Cancer Res 20:1735-1740

6. Nakano Y, Matsunaga H, Saita T, Masato M, Katano M, Okabe H (1998) Antiproliferative constituents in Umbelliferae plants II. Screening for polyacetylenes in some umbelliferae plants, and isolation of panaxynol and falcarindiol from the roots of Heracleum moellendorffii. Biol Pharm Bull 21:257-261

7. Fujioka T, Furumi K, Fujii H, Okabe H, Mihashi K, Nakano Y, Matsunaga H, Katano M, Masato M (1999) Antiproliferative constituents in Umbelliferae plants V. A new furanocoumarin and falcarindiol furanocoumarin ethers from the root of Angelica japonica. Chem Pharm Bull 47:96-100

8. Christensen LP, Brandt K (2006) Bioactive polyacetylenes in food plants of the Apiaceae family: occurrence, bioactivity and analysis. J Pharm Biomed Anal 41:683-693

9. Ikeda R, Nagao T, Okabe H, Nakano Y, Matsunaga H, Katano M, Mori M (1998) Antiproliferative constituents in Umbelliferae plants III. Constituents in the root and the ground part of $\mathrm{An}$ thriscus sylvestris Hoffm. Chem Pharm Bull 46:871-874

10. Ikeda R, Nagao T, Okabe H, Nakano Y, Matsunaga H, Katano M, Mori M (1998) Antiproliferative constituents in Umbelliferae plants IV. Constituents in the fruits of Anthriscus sylvestris Hoffm. Chem Pharm Bull 46:875-878 
11. Hartwell JL, Schrecker AW (1958) The chemistry of Podophyllum. Fortschr Chem Org Naturst 15:83-166

12. Lee KH, Xiao Z (2012) Podophyllotoxins and analogs. In: Cragg GM, Kingston DGI, Newman DJ (eds) Anticancer agents from natural products, 2nd edn. CRC press, Taylor \& Francis, New York, pp 95-122

13. Nagao T, Abe F, Okabe H (2001) Antiproliferative constituents in plants 7. Leaves of Clerodendron bungei and bark of C. trichotomum. Biol Pharm Bull 24:1338-1341

14. Abe F, Nagao T, Okabe H (2002) Antiproliferative constituents in plants 9. Aerial parts of Lippia dulcis and Lippia canescens. Biol Pharm Bull 25:920-922

15. Kinjo J, Nagao T, Okawa M, Nohara T, Yang CR, Nonaka G, Okabe H (2002) Antiproliferative activity of phenypropanoid ester glycosides from Ligstrum purpurascens. Nat Med 56:136-138

16. Saracoglu I, Harput U (2011) Phenylethanoid glycosides: naturally occurring apoptosis inducers. Planta Med. doi:10.1055/s0031-1282124

17. Kono S, Ikeda M, Tokudome S, Kuratsune M (1988) A casecontrol study of gastric cancer and diet in northern Kyushu, Japan. Jpn J Cancer Res 79:1064-1074

18. Kinjo J, Nagao T, Tanaka T, Nonaka G, Okabe H (2002) Activity-guided fractionation of green tea extract with antiproliferative activity against human stomach cancer cells. Biol Pharm Bull 25:1238-1240

19. Kinjo J, Nagao T, Tanaka T, Nonaka G, Okabe H (2001) Antiproliferative constituents in plants 8. Seeds of Rhynchosia volubilis. Biol Pharm Bull 24:1443-1445

20. Zhang YJ, Nagao T, Tanaka T, Nonaka G, Yang CR, Okabe H, Kouno I (2004) Antiproliferative activity of the main constituents from Phyllanthus emblica. Biol Pharm Bull 27:251-255

21. Khan HY, Zubair H, Ullah MF, Ahmad A, Hadi SM (2012) A prooxidant mechanism for the anticancer and chemopreventive properties of plant polyphenols. Curr Drug Targets 13:1738-1749

22. Nagao T, Abe F, Kinjo J, Okabe H (2002) Antiproliferative constituents in plants 10. Flavones from the leaves of Lantana montevidensis and consideration of structure-activity relationship. Biol Pharm Bull 25:875-879

23. Bible KC, Peethambaram PP, Oberg AL, Maples W, Groteluschen DL, Boente M, Burton JK, Gomez Dahl LC, Tibodeau JD, Isham CR, Maguire JL, Shridhar V, Kukla AK, Voll KJ, Mauer MJ, Colevas AD, Wright J, Doyle LA, Erlichman C (2012) A phase 2 trial of flavopiridol (Alvocidib) and cisplatin in platin-resistant ovarian and primary peritoneal carcinoma: MC0261. Gynecol Oncol 127:55-62

24. Konishi T, Shimada Y, Nagao T, Okabe H, Konoshima T (2002) Antiproliferative sesquiterpene lactones from the roots of Inula helenium. Biol Pharm Bull 25:1370-1372

25. Zhang S, Won YK, Ong CN, Shen HM (2005) Anti-cancer potential of sesquiterpene lactones: bioactivity and molecular mechanisms. Curr Med Chem Anticancer Agents 5:239-249

26. Fujioka T, Yoshida K, Fuji H, Nagao T, Okabe H, Mihashi K (2003) Antiproliferative constituents in Umbelliferae plants VI. New ursane-type saikosaponin analogs from the fruits of $\mathrm{Bu}$ pleurum rotundifolium. Chem Pharm Bull 51:365-372

27. Fujioka T, Yoshida K, Shibano H, Nagao T, Yoshida M, Matsunaga K, Takata J, Karube Y, Iwase Y, Okabe H, Mihashi K (2003) Antiproliferative constituents in Umbelliferae plants IX. New Triterpenoid glycosides from the fruits of Bupleurum rotundifolium. Chem Pharm Bull 54:1694-1704

28. Yoshida M, Fuchigami M, Nagao T, Okabe H, Matsunaga K, Takata J, Karube Y, Tsuchihashi R, Kinjo J, Mihashi K, Fujioka $\mathrm{T}$ (2005) Antiproliferative constituents in Umbelliferae plants VII. Active triterpenes and rosmarinic acid from Centella asiatica. Biol Pharm Bull 28:173-175
29. Zhang W, Men X, Lei P (2014) Review on anti-tumor effect of triterpene acid compounds. J Cancer Res Therapeutics 10:14-19

30. Chaya N, Terauchi K, Yamagata Y, Kinjo J, Okabe H (2004) Antiproliferative constituents in plants 14. Coumarins and acridone alkaroids from Boenninghausenia japonica. Biol Pharm Bull 27:1312-1316

31. Klenkar J, Molnar M (2015) Natural and synthetic coumarins as potential anticancer agents. J Chem Pharm Res 7:1223-1238

32. Nakano D, Ishitsuka $\mathrm{K}$, Hatsuse $\mathrm{T}$, Tsuchihashi R, Okawa $\mathrm{M}$, Okabe H, Tamura K, Kinjyo J (2011) Screening of promising chemotherapeutic candidates against human adult T-cell leukemia/lymphoma from plants: active principles structure-activity relationships with withanolides. J Nat Med 65:559-567

33. Nakano D, Ishitsuka K, Katsuya H, Kunami N, Nogami R, Yoshimura Y, Matsuda M, Kamikawa M, Tsuchihashi R, Okawa M, Ikeda T, Nohara T, Tamura K, Kinjo J (2013) Screening of promising chemotherapeutic candidates from plants against human adult T-cell leukemia/lymphoma (II): apoptosis of antiproliferactive principle (24,25-dihydrowithanolide D) against ATL cell lines and structure-activity relationships with withanolides isolated from solanaceous plants. J Nat Med 67:415-420

34. Yen CY, Chiu CC, Chang FR, Chen JYF, Hwang CC, Hseu YC, Yang HL, Lee AYL, Tsai MT, Guo ZL, Cheng YS, Liu YC, Lan YH, Chang YC, Ko YC, Chang HW, Wu YC (2010) 4 $\beta$-Hydroxywithanolide E from Physalis peruviana (golden berry) inhibits growth of human lung cancer cells through DNA damage, apoptosis and G2/M arrest. BMC Cancer 10:46. doi:10.1186/ 1471-2407-10-46

35. Mondal S, Mandal C, Sangwan R, Chandra S, Mandal C (2010) Withanolide D induces apoptosis in leukemia by targeting the activation of neutral sphingomyelinase-ceramide cascade mediated by synergistic activation of c-Jun N-terminal kinase and p38 mitogen-activated protein kinase. Mol Cancer 9:239. doi:10. 1186/1476-4598-9-239

36. Wang HC, Tsai YL, Wu YC, Chang FR, Liu MH, Chen WY, Wu CC (2012) Withanolides-induced breast cancer cell death is correlated with their ability to inhibit heat protein 90. PLoS One 7:e37764. doi:10.1371/journal.pone.0037764

37. Nakano D, Ishitsuka K, Kamikawa M, Matsuda M, Tsuchihashi R, Okawa M, Okabe H, Tamura K, Kinjo J (2013) Screening of promising chemotherapeutic candidates from plants against human adult T-cell leukemia/lymphoma (III). J Nat Med 67:894-903

38. Liu Y, Liu J, Di D, Li M, Fen Y (2013) Structural and mechanistic bases of the anticancer activity of natural aporphinoid alkaloids. Curr Topics Med Chem 13:2116-2126

39. Nakano D, Ishitsuka K, Ikeda M, Tsuchihashi R, Okawa M, Ikeda T, Nohara T, Tamura K, Kinjo J (2015) Screening of promising chemotherapeutic candidates from plants against human adult T-cell leukemia/lymphoma (IV): phenanthroindolizidine alkaloids from Tylophora tanakae leaves. J Nat Med 69:397-401

40. Dan S, Anne KL, Jette C, Boadan AB, Abe F, Jerzy WJ (2002) In vitro cytotoxic activity of phenanthroindolizidine alkaloids from Cynanchum vincetoxicum and Tylophora tanakae against drug-sensitive and multidrug-resistant cancer cells. J Nat Prod 65:1299-1302

41. Rao KN, Venkatachalam SR (2000) Inhibition of dihydrofolate reductase and cell growth activity by the phenanthroindolizidine alkaloids pergularinine and tylophorinidine: the in vitro cytotoxicity of these plant alkaloids and their potential as antimicrobial and anticancer agents. Toxicol In Vitro 14:53-59

42. Ueno S, Yamazaki R, Ikeda T, Yaegashi T, Matsuzaki T (2014) Antitumor effect of a novel phenanthroindolizidine alkaloid derivative through inhibition of protein synthesis. Anticancer Res 34:3391-3398 